\title{
ASYMPTOTIC EXPANSION OF A MULTISCALE NUMERICAL SCHEME FOR COMPRESSIBLE MULTIPHASE FLOW
}

\author{
RÉMI ABGRALL* AND VINCENT PERRIER ${ }^{\dagger}$
}

\begin{abstract}
The simulation of compressible multiphase problems is a difficult task for modelization and mathematical reasons. Here, thanks to a probabilistic multiscale interpretation of multiphase flows, we construct a numerical scheme that provides a solution to these difficulties. Three types of terms can be identified in the scheme in addition to the temporal term. One is a conservative term, the second one plays the role of a nonconservative term that is related to interfacial quantities, and the last one is a relaxation term that is associated with acoustic phenomena. The key feature of the scheme is that it is locally conservative, contrarily to many other schemes devoted to compressible multiphase problems. In many physical situations, it is reasonable to assume that the relaxation is instantaneous. We present an asymptotic expansion of the scheme that keeps the local conservation properties of the original scheme. The asymptotic expansion relies on the understanding of an equilibrium variety. Its structure depends, in principle, on the Riemann solver. We show that it is not the case for several standard solvers, and hence this variety is characterized by the local pressure and velocity of the flow. Several numerical test cases are presented in order to demonstrate the potential of this technique.
\end{abstract}

1. Introduction. In [10], we have considered the following model for compressible multiphase flows:

$$
\begin{array}{rlrl}
\frac{\partial \alpha^{(k)}}{\partial t} & +\mathbf{u}_{I} \cdot \nabla \alpha^{(k)} & =\mu\left(P^{(k)}-P^{(\bar{k})}\right), \\
\frac{\partial\left(\alpha^{(k)} \rho^{(k)}\right)}{\partial t}+\operatorname{div}\left(\alpha^{(k)} \rho^{(k)} \mathbf{u}^{(k)}\right) & =0, \\
\frac{\partial\left(\alpha^{(k)} \rho^{(k)} \mathbf{u}^{(k)}\right)}{\partial t}+\operatorname{div}\left(\alpha^{(k)} \rho^{(k)} \mathbf{u}^{(k)} \otimes \mathbf{u}^{(k)}\right)+\nabla\left(\alpha^{(k)} P^{(k)}\right) & =P_{I} \nabla \alpha^{(k)} \\
& +\lambda\left(\mathbf{u}^{(\bar{k})}-\mathbf{u}^{(k)}\right), \\
\frac{\partial\left(\alpha^{(k)} \rho^{(k)} E^{(k)}\right)}{\partial t}+\operatorname{div}\left(\alpha^{(k)}\left(\rho^{(k)} E^{(k)}+P^{(k)}\right) \mathbf{u}^{(k)}\right) & =P_{I} \mathbf{u}_{I} \cdot \nabla \alpha^{(k)} \\
& -\mu P_{I}\left(P^{(k)}-P^{(\bar{k})}\right) \\
& +\lambda \mathbf{u}_{I}\left(\mathbf{u}^{(\bar{k})}-\mathbf{u}^{(k)}\right) .
\end{array}
$$

In (1.1), $P^{(k)}$ is the pressure of phase $\Sigma_{k}$, and the total energy is related to the pressure by $E^{(k)}=\varepsilon^{(k)}\left(P^{(k)}, \rho^{(k)}\right)+\frac{1}{2} \mathbf{u}^{(k)^{2}}$ where the internal energy is a concave function of its arguments. We define $\bar{k}=1$ (resp., $=2$ ) when $k=2$ (resp., $k=1$ ). The interface velocity and pressure $\mathbf{u}_{I}$ and $P_{I}$ are modeled, and this is one of the difficult points; see, e.g., Drew and Passman [4]. The variables $\mu$ and $\lambda$ are relaxation parameters that depend on the interfacial area.

With respect to standard approaches, the seven equation model (1.1) has several original features and properties. First, (1.1) is hyperbolic whatever the choice of the interface parameters. This is in contrast with models that are only conditionally hyperbolic such as in $[12,5]$. From the numerical point of view, the interesting consequence is that one may adapt modern standard techniques for the approximation

* Mathématiques Appliquées de Bordeaux, 33405 Talence Cedex, France, and Institut Universitaire de France (abgrall@math.u-bordeaux1.fr).

† Mathématiques Appliquées de Bordeaux, 33405 Talence Cedex, France, and Centre des Lasers Intenses et Applications, 33405 Talence Cedex, France (perrier@math.u-bordeaux1.fr). 
of hyperbolic problems. Another interesting feature is the occurrence of the relaxation parameters $\mu$ and $\lambda$ that can be interpreted as a way to introduce the local flow topology.

However, the model (1.1) leads to several difficult issues:

- A modelization problem. How can we define the interface velocity and pressure? From a rigorous point of view, the situation is rather clear only when $\lambda \rightarrow+\infty$ and $\mu \rightarrow+\infty$, in which case we have pressure and velocity equilibrium. This corresponds to interface problems or problems where the phases are intimately mixed.

- A mathematical problem. The system cannot be cast into conservative form, which induces difficulties for the definition of a shock and for the derivation of a numerical scheme. This point is in contradiction with the initial goal, the use of standard hyperbolic techniques for the simulation of multiphase flow problems.

In the limit $\lambda, \mu \rightarrow+\infty$, while $\mu / \lambda$ remains bounded, Murrone and Guillard [8] have obtained by asymptotic expansion the following model:

$$
\begin{array}{ll}
\frac{\partial \alpha^{(k)}}{\partial t}+\mathbf{u} \cdot \nabla \alpha^{(k)} & =\frac{\rho^{(\bar{k})} a^{(\bar{k})^{2}}-\rho^{(k)} a^{(k)^{2}}}{\frac{\rho^{(k)} a^{(k)^{2}}}{\alpha^{(k)}}+\frac{\rho^{(\bar{k})} a^{(\bar{k})^{2}}}{\alpha^{(k)}}} \operatorname{div} \mathbf{u}, \\
\frac{\partial\left(\alpha^{(k)} \rho^{(k)}\right)}{\partial t}+\operatorname{div}\left(\alpha^{(k)} \rho^{(k)} \mathbf{u}\right) & =0 \\
\frac{\partial(\rho \mathbf{u})}{\partial t}+\operatorname{div}(\rho \mathbf{u} \otimes \mathbf{u})+\nabla P & =0 \\
\frac{\partial(\rho E)}{\partial t}+\operatorname{div}((\rho E+P) \mathbf{u}) & =0,
\end{array}
$$

where $\rho$ and $E$ are the mixture density and the mixture energy defined by

$$
\begin{gathered}
\rho=\alpha^{(1)} \rho^{(1)}+\alpha^{(2)} \rho^{(2)} \\
E=\frac{\alpha^{(1)} \rho^{(1)} E^{(1)}+\alpha^{(2)} \rho^{(2)} E^{(2)}}{\rho}
\end{gathered}
$$

and $a^{(k)}$ is the isentropic sound velocity of each phase defined by

$$
a^{(k)^{2}}=\left(\frac{\partial P^{(k)}}{\partial \rho^{(k)}}\right)_{s^{(k)}} .
$$

This system was obtained by developing the different pressures and velocities of (1.1) around the equilibrium variables $P$ and $\mathbf{u}$. In what follows, we often denote this model as the "five equations model."

This system can be shown to be unconditionally hyperbolic. Unfortunately, a nonconservative product appears one more time in the volume of fluid equation. This term can be interpreted as follows: when a shock enters a mixture zone, the volume fraction must change because the shock speed is different in the two phases. This term is proportional to the divergence of the velocity and does not play any role in pure interface problems. In contrast, this term does not vanish in general across a shock wave, and it induces a change in volume. However, the difficulty, from a mathematical 
point of view, is that this term has no precise meaning in the case where $\mathbf{u}$ and

$$
\frac{\rho^{(\bar{k})} a^{(\bar{k})^{2}}-\rho^{(k)} a^{(k)^{2}}}{\frac{\rho^{(k)} a^{(k)}}{\alpha^{(k)}}+\frac{\rho^{(\bar{k})} a^{(\bar{k})^{2}}}{\alpha^{(k)}}}
$$

are simultaneously discontinuous. In that case, it is difficult to derive a numerical scheme.

From Murrone and Guillard analysis, it is clear that something has to be added in the volume of fluid equation to take into account the volume changes across nonlinear waves. In the present contribution, we propose to answer this problem by starting with the scheme of [2] and then to derive an asymptotic expansion from the discrete scheme, instead of the system of PDE (1.1). The scheme of [2], which is well adapted to approximate multiphase problems, relies on a multiscale representation of the flow. We recall the fact that (1.1) is obtained from an ensemble average of realization; for any realization we have well-localized interfaces between phases, with possibly different scales for bubbles. From the numerical point of view, we write a scheme for each realization, using standard assumptions in the finite volume context. This scheme makes use of one's favorite Riemann solver. Here we consider Godunov, HLL type, acoustic, and relaxation solvers. We take into account the various scales by dividing cells into subcells being filled by any of the two phases. Then we make an ensemble average of the different schemes. This avoids defining precisely interface variables (even though a limit model can be derived), and this also solves the problem of nonconservative products (since the scheme for each realization is obtained from conservation principles). We can identify terms that correspond to relaxation terms as in (1.1); they are the consequence of acoustic effects. The last step, writing the scheme for (1.2), is obtained by doing an asymptotic expansion of the scheme of [2].

This paper is organized as follows. We first recall in detail the results of [2] with a particular emphasis on the relaxation terms. This is the topic of section 2 . The structure of the equilibrium variety for several numerical schemes is studied in section 3. Section 4 is devoted to the derivation of the asymptotic scheme of (1.2). Numerical illustrations are provided in section 5 .

2. A numerical scheme for the seven equations model. The derivation of the scheme uses the ensemble averaging ideas of Drew and Passman [4] combined with the discretization principle introduced by Godunov. In [4], the nonconservative and the modelization terms appear after an ensemble average. The idea of [2] is to begin the derivation of the numerical scheme before the averaging procedure. We describe the scheme in the case of the Godunov solver. All this can easily be adapted to other solvers. We also note that the different terms of (2.3) can be approximated with different solvers; the only constraint is conservation.

At each time step, the flow is described in each computational cell by the average $W_{j}=\left(\alpha_{j}^{(1)}, \alpha_{j}^{(1)} \rho_{j}^{(1)}, \alpha_{j}^{(1)} \rho_{j}^{(1)} u_{j}^{(1)}, \alpha_{j}^{(1)} \rho_{j}^{(1)} E_{j}^{(1)}, \alpha_{j}^{(2)}, \alpha_{j}^{(2)} \rho_{j}^{(2)}, \alpha_{j}^{(2)} \rho_{j}^{(2)} u_{j}^{(2)}, \alpha_{j}^{(2)} \rho_{j}^{(2)} E_{j}^{(2)}\right)$.

We consider a family of random subdivision of the cell

$$
\mathcal{C}_{j}=\left[x_{j-1 / 2}, x_{j+1 / 2}\right]=\bigcup_{k}\left[\xi_{k}, \xi_{k+1}\right] .
$$

In each of the subcells $] \xi_{k}, \xi_{k+1}$ [, we randomly set the flow variables (see Figure 1)

$$
U_{j}^{(1)}=\left(\rho_{j}^{(1)}, \rho_{j}^{(1)} u_{j}^{(1)}, \rho_{j}^{(1)} E_{j}^{(1)}\right) \quad \text { or } \quad U_{j}^{(2)}=\left(\rho_{j}^{(2)}, \rho_{j}^{(2)} u_{j}^{(2)}, \rho_{j}^{(2)} E_{j}^{(2)}\right) .
$$




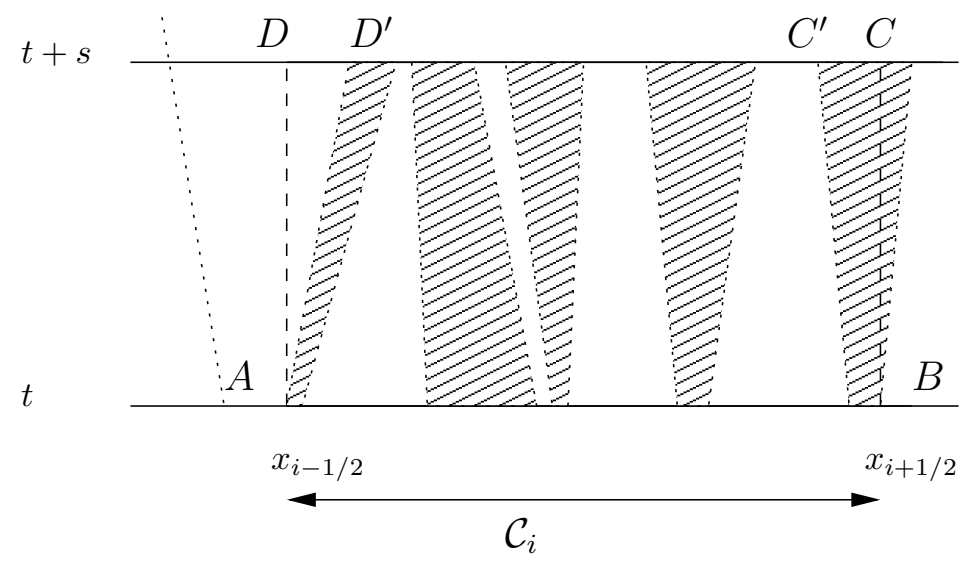

FIG. 1. Evolution of each phase after a random subdivision of the cell $\mathcal{C}_{i}$ between time $t$ and $t+s$.

The only constraint on the random subdivision is that it must be consistent with the volume fraction of each phase. Therefore, if $X$ is the indicator function of $\Sigma_{1}$, the average $\mathcal{E}\left(\int_{x_{j-1 / 2}}^{x_{j+1 / 2}} X d x\right)$ must precisely be $\alpha_{1}^{(j)}\left(x_{j+1 / 2}-x_{j-1 / 2}\right)$.

In the cell $\mathcal{C}_{i}$, the phase $\Sigma_{1}$ satisfies

$$
\int_{\mathcal{C}_{i}} X\left(\frac{\partial \mathbf{U}}{\partial t}+\frac{\partial \mathbf{F}}{\partial x}\right) \mathrm{d} x \mathrm{~d} t=0
$$

Then

$$
\sum_{j=0}^{N(\omega)-1} \int_{\xi_{j}}^{\xi_{j+1}} X \frac{\partial \mathbf{U}}{\partial t} \mathrm{~d} x+\sum_{j=0}^{N(\omega)-1} \int_{\xi_{j}}^{\xi_{j+1}} X \frac{\partial \mathbf{F}}{\partial x} \mathrm{~d} x=0
$$

and the characteristic function $X$ obeys

$$
\sum_{j=0}^{N(\omega)-1} \int_{t}^{t+s} \frac{\partial X}{\partial t} \mathrm{~d} x \mathrm{~d} t+\sum_{j=0}^{N(\omega)-1} \int_{t}^{t+s} \int_{\xi_{j}}^{\xi_{j+1}} \sigma \frac{\partial X}{\partial x} \mathrm{~d} x \mathrm{~d} t=0
$$

where $\sigma$ is the local interface velocity. We denote by $\lambda_{\max }$ the maximum wave speed in the internal Riemann problems. Under the CFL condition

$$
\left|\lambda_{\max }\right| \frac{s}{\Delta \xi} \leq \frac{1}{2}
$$

we can integrate $(2.1)$ to get

$$
\sum_{l=1}^{N(\omega)-1} \int_{t}^{t+s} \int_{\xi_{l}}^{\xi_{l+1}} X \frac{\partial \mathbf{U}}{\partial t} \mathrm{~d} x \mathrm{~d} t+\sum_{l=1}^{N(\omega)-1} \int_{t}^{t+s} \int_{\xi_{l}}^{\xi_{l+1}} X \frac{\partial \mathbf{F}}{\partial x} \mathrm{~d} x \mathrm{~d} t=0 .
$$

Equation (2.2) can be split into three parts: interaction with the left cell (2.3a), interaction with the right cell $(2.3 \mathrm{c})$, and internal interactions $(2.3 \mathrm{~b})$ :

$$
\int_{A D^{\prime} D} X\left(\frac{\partial \mathbf{U}}{\partial t}+\frac{\partial \mathbf{F}}{\partial x}\right) \mathrm{d} x \mathrm{~d} t
$$




$$
\begin{aligned}
+\sum_{l=2}^{N(\omega)-1} \int_{t}^{t+s} \int_{\xi_{l}+s^{\prime} \sigma\left(\mathbf{U}_{i}^{l-1}, \mathbf{U}_{i}^{l}\right)}^{\xi_{l+1}+s^{\prime} \sigma\left(\mathbf{U}_{i}^{l}, \mathbf{U}_{i}^{l+1}\right)} & X\left(\frac{\partial \mathbf{U}}{\partial t}+\frac{\partial \mathbf{F}}{\partial x}\right) \mathrm{d} x \mathrm{~d} s^{\prime} \\
& +\int_{C B B^{\prime}} X\left(\frac{\partial \mathbf{U}}{\partial t}+\frac{\partial \mathbf{F}}{\partial x}\right) \mathrm{d} x \mathrm{~d} t=0 .
\end{aligned}
$$

We study the three terms of (2.3).

- Boundary terms. For the term (2.3a), a straightforward calculation provides

$$
\begin{aligned}
\int_{A D^{\prime} D} X\left(\frac{\partial \mathbf{U}}{\partial t}+\frac{\partial \mathbf{F}}{\partial x}\right) \mathrm{d} x \mathrm{~d} t= & \int_{A D^{\prime} D}\left(\frac{\partial X \mathbf{U}}{\partial t}+\frac{\partial X \mathbf{F}}{\partial x}\right) \mathrm{d} x \mathrm{~d} t \\
& -\int_{A D^{\prime} D}\left(\mathbf{U} \frac{\partial X}{\partial t}+\mathbf{F}(\mathbf{U}) \frac{\partial X}{\partial x}\right) \mathrm{d} x \mathrm{~d} t \\
= & \int_{x_{i-\frac{1}{2}}}^{x_{i-\frac{1}{2}}+s \sigma^{+}\left(\mathbf{U}_{i-1}^{+}, \mathbf{U}_{i}^{-}\right)} X(x, t+s) \mathbf{U}(x, t+s) \mathrm{d} x \\
& -s X\left(x_{i-\frac{1}{2}}, t^{+}\right) \mathbf{F}\left(\mathbf{U}_{i-\frac{1}{2}}^{\star}\right) \\
& +s \mathbf{F}^{l a g}\left(\mathbf{U}_{i-1}^{+}, \mathbf{U}_{i}^{-}\right)[X]_{j=0} .
\end{aligned}
$$

Doing the same for the right term (2.3c), we find

$$
\begin{aligned}
\int_{B C C^{\prime}} X\left(\frac{\partial \mathbf{U}}{\partial t}+\frac{\partial \mathbf{F}}{\partial x}\right) \mathrm{d} x \mathrm{~d} t= & \int_{x_{i+\frac{1}{2}}}^{x_{i+\frac{1}{2}}+s \sigma^{-}\left(\mathbf{U}_{i}^{+}, \mathbf{U}_{i+1}^{-}\right)} X(x, t+s) \mathbf{U}(x, t+s) \mathrm{d} x \\
& -s X\left(x_{i+\frac{1}{2}}, t^{+}\right) \mathbf{F}\left(\mathbf{U}_{i+\frac{1}{2}}^{\star}\right) \\
& +s \mathbf{F}^{l a g}\left(\mathbf{U}_{i}^{+}, \mathbf{U}_{i+1}^{-}\right)[X]_{j=N(\omega)} .
\end{aligned}
$$

- Internal terms (2.3b). A similar algebra provides

$$
\begin{aligned}
\int_{t}^{t+s} & \int_{\xi_{l}+s^{\prime} \sigma\left(\mathbf{U}_{i}^{l-1}, \mathbf{U}_{i}^{l}\right)}^{\xi_{l+1}+s^{\prime} \sigma\left(\mathbf{U}_{i}^{l}, \mathbf{U}_{i}^{l+1}\right)} X\left(\frac{\partial \mathbf{U}}{\partial t}+\frac{\partial \mathbf{F}}{\partial x}\right) \mathrm{d} x \mathrm{~d} s^{\prime} \\
= & s\left([X]_{j} \mathbf{F}^{l a g}\left(\mathbf{U}_{i}^{j}, \mathbf{U}_{i}^{j+1}\right)+[X]_{j-1} \mathbf{F}^{l a g}\left(\mathbf{U}_{i}^{j-1}, \mathbf{U}_{i}^{j}\right)\right) \\
& +\int_{\xi_{j}+s \sigma\left(\mathbf{U}_{i}^{j-1}, \mathbf{U}_{i}^{j}\right)}^{\xi_{j+1}+s \sigma\left(\mathbf{U}_{i}^{j}, \mathbf{U}_{i}^{j+1}\right)} X(x, t+s) \mathbf{U}(x, t+s) \mathrm{d} x \\
& -\int_{\xi_{j}}^{\xi_{j+1}} X(x, t) \mathbf{U}(x, t) \mathrm{d} x .
\end{aligned}
$$

By summing the different terms and taking the limit when $s \rightarrow 0$, we obtain the 
semidiscrete scheme

$$
\begin{aligned}
\frac{\partial}{\partial t}\left(\frac{1}{\Delta x} \int_{x_{i-\frac{1}{2}}}^{x_{i+\frac{1}{2}}} X(x, t) \mathbf{U}(x, t) \mathrm{d} x\right) \\
\quad+\frac{1}{\Delta x}\left(X\left(x_{i+\frac{1}{2}}, t^{+}\right) \mathbf{F}\left(\mathbf{U}_{i+\frac{1}{2}}^{\star}\right)-X\left(x_{i-\frac{1}{2}}, t^{+}\right) \mathbf{F}\left(\mathbf{U}_{i-\frac{1}{2}}^{\star}\right)\right) \\
=\frac{1}{\Delta x} \sum_{j=1}^{N(\omega)}\left([X]_{j} \mathbf{F}^{\text {lag }}\left(\mathbf{U}_{i}^{j}, \mathbf{U}_{i}^{j-1}\right)-[X]_{j-1} \mathbf{F}^{\text {lag }}\left(\mathbf{U}_{i}^{j-1}, \mathbf{U}_{i}^{j-2}\right)\right) \\
\quad+\frac{1}{\Delta x}\left([X]_{0} \mathbf{F}^{\text {lag }}\left(\mathbf{U}_{i-1}^{+}, \mathbf{U}_{i}^{-}\right)+[X]_{N(\omega)} \mathbf{F}^{\text {lag }}\left(\mathbf{U}_{i}^{+}, \mathbf{U}_{i+1}^{-}\right)\right) .
\end{aligned}
$$

We may assume that two adjacent subcells contains different phases, ${ }^{1}$ so that the sum in (2.4) can be rewritten as

$$
\begin{array}{r}
\sum_{j=1}^{N(\omega)-1}\left([X]_{j} \mathbf{F}^{\text {lag }}\left(\mathbf{U}_{i}^{j}, \mathbf{U}_{i}^{j-1}\right)-[X]_{j-1} \mathbf{F}^{\text {lag }}\left(\mathbf{U}_{i}^{j-1}, \mathbf{U}_{i}^{j-2}\right)\right) \\
= \pm N(\omega)_{i}\left(\mathbf{F}^{\text {lag }}\left(\mathbf{U}_{i}^{(2)}, \mathbf{U}_{i}^{(1)}\right)-\mathbf{F}^{\text {lag }}\left(\mathbf{U}_{i}^{(1)}, \mathbf{U}_{i}^{(2)}\right)\right) .
\end{array}
$$

2.1. Averaging procedure. It remains to take the mathematical expectancy of the semidiscrete scheme (2.4). For the first term, we have

$$
\mathcal{E}\left(\frac{\partial}{\partial t}\left(\frac{1}{\Delta x} \int_{x_{i-\frac{1}{2}}}^{x_{i+\frac{1}{2}}} X(x, t) \mathbf{U}(x, t) \mathrm{d} x\right)\right)=\frac{\partial\left(\alpha_{i}^{(1)} \mathbf{U}_{i}^{(1)}\right)}{\partial t} .
$$

Then the scheme can be rewritten as

$$
\begin{gathered}
\frac{\partial\left(\alpha_{i}^{(1)} \mathbf{U}_{i}^{(1)}\right)}{\partial t}+\frac{1}{\Delta x}\left(\mathcal{E}\left(X\left(x_{i+\frac{1}{2}}, t^{+}\right) \mathbf{F}\left(\mathbf{U}_{i+\frac{1}{2}}^{\star}\right)\right)-\mathcal{E}\left(X\left(x_{i-\frac{1}{2}}, t^{+}\right) \mathbf{F}\left(\mathbf{U}_{i-\frac{1}{2}}^{\star}\right)\right)\right) \\
=\lambda_{i}\left(\mathbf{F}^{\ell a g}\left(\mathbf{U}_{i}^{(2)}, \mathbf{U}_{i}^{(1)}\right)-\mathbf{F}^{\ell a g}\left(\mathbf{U}_{i}^{(1)}, \mathbf{U}_{i}^{(2)}\right)\right) \\
\quad+\frac{1}{\Delta x}\left(\mathcal{E}\left([X]_{0}\right) \mathbf{F}^{\ell a g}\left(\mathbf{U}_{i-1}^{+}, \mathbf{U}_{i}^{-}\right)+\mathcal{E}\left([X]_{N(\omega)}\right) \mathbf{F}^{\ell a g}\left(\mathbf{U}_{i}^{+}, \mathbf{U}_{i+1}^{-}\right)\right)
\end{gathered}
$$

Here we have introduced the notation

$$
\lambda_{i}=\mathcal{E}\left(\frac{N(\omega)_{i}}{\Delta x}\right)
$$

which can be interpreted as the number of interfaces per cell. ${ }^{2}$

\footnotetext{
${ }^{1}$ Otherwise, if two neighboring cells contain the same phase, we gather them: this is not an extra assumption.

${ }^{2}$ In the multidimensional case, more than one relaxation parameter can be introduced. Typically, the same arguments show that there is one relaxation parameter associated with the pressure, two for the velocity in two dimensions, and three in three dimensions. These relaxation parameters depend on thermodynamical parameters, such as the acoustic impedance, but also on the interfacial area between the faces. More details can be found in [1]
} 
TABLE 1

Flux indicator for the Eulerian flux where we denote $\beta_{i+\frac{1}{2}}^{(l, p)}=\operatorname{sign}\left(\sigma\left(U_{i}^{l}, U_{i+1}^{p}\right)\right)$.

\begin{tabular}{|c|c|c|}
\hline Flow patterns & Left and right states & Flux indicator \\
\hline$\Sigma_{1}-\Sigma_{2}$ & $U_{i}^{(1)}, U_{i+1}^{(2)}$ & $\left(\beta_{i+\frac{1}{2}}^{(1,2)}\right)^{+}$ \\
$\Sigma_{1}-\Sigma_{1}$ & $U_{i}^{(1)}, U_{i+1}^{(1)}$ & 1 \\
$\Sigma_{2}-\Sigma_{1}$ & $U_{i}^{(2)}, U_{i+1}^{(1)}$ & $\left(-\beta_{i+\frac{1}{2}}^{(2,1)}\right)^{+}$ \\
$\Sigma_{2}-\Sigma_{2}$ & $U_{i}^{(2)}, U_{i+1}^{(2)}$ & 0 \\
\hline
\end{tabular}

Similarly we get, for $\Sigma_{2}$,

$(2.6)$

$$
\begin{aligned}
\frac{\partial\left(\alpha_{i}^{(2)} \mathbf{U}_{i}^{(2)}\right)}{\partial t}+\frac{1}{\Delta x}\left(\mathcal{E}\left(X\left(x_{i+\frac{1}{2}}, t^{+}\right) \mathbf{F}\left(\mathbf{U}_{i+\frac{1}{2}}^{\star}\right)\right)-\mathcal{E}\left(X\left(x_{i-\frac{1}{2}}, t^{+}\right) \mathbf{F}\left(\mathbf{U}_{i-\frac{1}{2}}^{\star}\right)\right)\right) \\
=-\lambda_{i}\left(\mathbf{F}^{\ell a g}\left(\mathbf{U}_{i}^{(2)}, \mathbf{U}_{i}^{(1)}\right)-\mathbf{F}^{\ell a g}\left(\mathbf{U}_{i}^{(1)}, \mathbf{U}_{i}^{(2)}\right)\right) \\
\quad-\frac{1}{\Delta x}\left(\mathcal{E}\left([X]_{0}\right) \mathbf{F}^{\ell a g}\left(\mathbf{U}_{i-1}^{+}, \mathbf{U}_{i}^{-}\right)+\mathcal{E}\left([X]_{N(\omega)}\right) \mathbf{F}^{\ell a g}\left(\mathbf{U}_{i}^{+}, \mathbf{U}_{i+1}^{-}\right)\right)
\end{aligned}
$$

In (2.5) and (2.6), the expected fluxes $\mathcal{E}\left(X\left(x_{i \pm \frac{1}{2}}, t^{+}\right) \mathbf{F}\left(\mathbf{U}_{i+\frac{1}{2}}^{\star}\right)\right), \mathcal{E}\left([X]_{0}\right)$ $\mathbf{F}^{\ell a g}\left(\mathbf{U}_{i-1}^{+}, \mathbf{U}_{i}^{-}\right)$, and $\mathcal{E}\left([X]_{N(\omega)}\right) \mathbf{F}^{\ell a g}\left(\mathbf{U}_{i}^{+}, \mathbf{U}_{i+1}^{-}\right)$are evaluated by a close examination of each possible case.

In the case of $\mathcal{E}\left(X\left(x_{i \pm \frac{1}{2}}, t^{+}\right) \mathbf{F}\left(\mathbf{U}_{i+\frac{1}{2}}^{\star}\right)\right)$, and specializing on the right interface of the cell $\mathcal{C}_{i}$, four cases may happen:

- either fluid $\Sigma_{1}$ is on the left and fluid $\Sigma_{1}$ is on the right

- or fluid $\Sigma_{1}$ is on the left and fluid $\Sigma_{2}$ is on the right

- or fluid $\Sigma_{2}$ is on the left and fluid $\Sigma_{2}$ is on the right

- or fluid $\Sigma_{2}$ is on the left and fluid $\Sigma_{1}$ is on the right.

Depending on which case occurs, we may or may not take into account the fluxes. Four fluxes are evaluated (and summarized in Table 1); they are flagged when needed following the procedure we describe now. Of course, a similar situation happens on the left interface. Then if we define

$$
\begin{aligned}
& \mathcal{P}_{i+\frac{1}{2}}\left(\Sigma_{1}, \Sigma_{1}\right)=\mathcal{P}\left(X\left(x_{i+\frac{1}{2}}^{-}\right)=1 \text { and } X\left(x_{i+\frac{1}{2}}^{+}\right)=1\right), \\
& \mathcal{P}_{i+\frac{1}{2}}\left(\Sigma_{2}, \Sigma_{2}\right)=\mathcal{P}\left(X\left(x_{i+\frac{1}{2}}^{-}\right)=0 \text { and } X\left(x_{i+\frac{1}{2}}^{+}\right)=0\right), \\
& \mathcal{P}_{i+\frac{1}{2}}\left(\Sigma_{1}, \Sigma_{2}\right)=\mathcal{P}\left(X\left(x_{i+\frac{1}{2}}^{-}\right)=1 \text { and } X\left(x_{i+\frac{1}{2}}^{+}\right)=0\right), \\
& \mathcal{P}_{i+\frac{1}{2}}\left(\Sigma_{2}, \Sigma_{1}\right)=\mathcal{P}\left(X\left(x_{i+\frac{1}{2}}^{-}\right)=0 \text { and } X\left(x_{i+\frac{1}{2}}^{+}\right)=1\right),
\end{aligned}
$$


Flux indicator for the Lagrangian flux where we denote $\beta_{i+\frac{1}{2}}^{(l, p)}=\operatorname{sign}\left(\sigma\left(U_{i}^{l}, U_{i+1}^{p}\right)\right)$.

\begin{tabular}{|c|c|c|}
\hline Flow patterns & Lagrangian flux & Flux indicator \\
\hline$\Sigma_{1}-\Sigma_{2}$ & $F^{\ell a g}\left(U_{i}^{(1)}, U_{i+1}^{(2)}\right)$ & $\left(\beta_{i+\frac{1}{2}}^{(1,2)}\right)^{-}$ \\
$\Sigma_{1}-\Sigma_{1}$ & $F^{\ell a g}\left(U_{i}^{(1)}, U_{i+1}^{(1)}\right)$ & 0 \\
$\Sigma_{2}-\Sigma_{1}$ & $F^{\ell a g}\left(U_{i}^{(2)}, U_{i+1}^{(1)}\right)$ & $-\left(-\beta_{i+\frac{1}{2}}^{(2,1)}\right)^{-}$ \\
$\Sigma_{2}-\Sigma_{2}$ & $F^{\ell a g}\left(U_{i}^{(2)}, U_{i+1}^{(2)}\right)$ & 0 \\
\hline
\end{tabular}

we get

$$
\begin{aligned}
\mathcal{E}\left(X\left(x_{i+\frac{1}{2}}, t_{n}^{+}\right) \mathbf{F}\left(U_{i+\frac{1}{2}}^{\star}\right)\right)=\mathcal{P}_{i+\frac{1}{2}}\left(\Sigma_{1}, \Sigma_{1}\right) \mathbf{F}\left(\mathbf{U}_{i}^{(1)}, \mathbf{U}_{i+1}^{(1)}\right) \\
+\mathcal{P}_{i+\frac{1}{2}}\left(\Sigma_{1}, \Sigma_{2}\right)\left(\beta_{i+\frac{1}{2}}^{(1,2)}\right)^{+} \mathbf{F}\left(\mathbf{U}_{i}^{(1)}, \mathbf{U}_{i+1}^{(2)}\right) \\
+\mathcal{P}_{i+\frac{1}{2}}\left(\Sigma_{2}, \Sigma_{1}\right)\left(-\beta_{i+\frac{1}{2}}^{(2,1)}\right)^{+} \mathbf{F}\left(\mathbf{U}_{i}^{(2)}, \mathbf{U}_{i+1}^{(1)}\right) .
\end{aligned}
$$

Similarly, in the case of the Lagrangian term of the right interface, the four cases are summarized in Table 2; a similar situation occurs at the left interface. We get for the right interface

$$
\begin{aligned}
\mathcal{E}\left([X]_{N(\omega)}\right) \mathbf{F}^{\ell a g}\left(\mathbf{U}_{i}^{N(\omega)}, \mathbf{U}_{i+1}^{-}\right)= & \mathcal{P}_{i+\frac{1}{2}}\left(\Sigma_{1}, \Sigma_{2}\right)\left(\beta_{i+\frac{1}{2}}^{(1,2)}\right)^{-} \mathbf{F}^{\ell a g}\left(\mathbf{U}_{i}^{(1)}, \mathbf{U}_{i+1}^{(2)}\right) \\
& -\mathcal{P}_{i+\frac{1}{2}}\left(\Sigma_{2}, \Sigma_{1}\right)\left(\beta_{i+\frac{1}{2}}^{(2,1)}\right)^{-} \mathbf{F}^{\ell a g}\left(\mathbf{U}_{i}^{(2)}, \mathbf{U}_{i+1}^{(1)}\right)
\end{aligned}
$$

and for the left one

$$
\begin{aligned}
\mathcal{E}\left([X]_{0}\right) \mathbf{F}^{\ell a g}\left(\mathbf{U}_{i-1}^{+}, \mathbf{U}_{i}^{0}\right)= & -\mathcal{P}_{i-\frac{1}{2}}\left(\Sigma_{1}, \Sigma_{2}\right)\left(\beta_{i-\frac{1}{2}}^{(1,2)}\right)^{+} \mathbf{F}^{\ell a g}\left(\mathbf{U}_{i}^{(1)}, \mathbf{U}_{i+1}^{(2)}\right) \\
& +\mathcal{P}_{i-\frac{1}{2}}\left(\Sigma_{2}, \Sigma_{1}\right)\left(\beta_{i-\frac{1}{2}}^{(2,1)}\right)^{+} \mathbf{F}^{\ell a g}\left(\mathbf{U}_{i-1}^{(2)}, \mathbf{U}_{i}^{(1)}\right) .
\end{aligned}
$$

These expressions are given for the phase $\Sigma_{1}$. Similar expressions can be given for $\Sigma_{2}$ : the variable $X$ is changed into $1-X$, and the phase index for $\Sigma_{1}$ (resp., $\Sigma_{2}$ ) is changed into the one for $\Sigma_{2}$ (resp., $\Sigma_{1}$ ).

2.2. A numerical scheme. As detailed in [2], a natural choice for the probability is

$$
\begin{aligned}
& \mathcal{P}_{i+\frac{1}{2}}\left(\Sigma_{1}, \Sigma_{1}\right)=\min \left(\alpha_{i}^{(1)}, \alpha_{i+1}^{(1)}\right), \\
& \mathcal{P}_{i+\frac{1}{2}}\left(\Sigma_{2}, \Sigma_{2}\right)=\min \left(\alpha_{i}^{(2)}, \alpha_{i+1}^{(2)}\right), \\
& \mathcal{P}_{i+\frac{1}{2}}\left(\Sigma_{1}, \Sigma_{2}\right)=\max \left(\alpha_{i}^{(1)}-\alpha_{i+1}^{(1)}, 0\right), \\
& \mathcal{P}_{i+\frac{1}{2}}\left(\Sigma_{2}, \Sigma_{1}\right)=\max \left(\alpha_{i}^{(2)}-\alpha_{i+1}^{(2)}, 0\right) .
\end{aligned}
$$


This leads to the following numerical scheme:

$$
\begin{array}{r}
\frac{\partial\left(\alpha^{(1)} \mathbf{U}^{(1)}\right)}{\partial t}+\frac{\mathcal{E}(X \mathbf{F})_{i+\frac{1}{2}}-\mathcal{E}(X \mathbf{F})_{i-\frac{1}{2}}}{\Delta x} \\
=\frac{1}{\Delta x} \mathcal{E}\left(\mathbf{F}^{\ell a g} \frac{\partial X}{\partial x}\right)_{i, \text { bound }}+\mathcal{E}\left(\mathbf{F}^{\ell a g} \frac{\partial X}{\partial x}\right)_{i, \text { relax }}
\end{array}
$$

with

$$
\begin{aligned}
\mathcal{E}(X \mathbf{F})_{i+\frac{1}{2}}=\max & \left(\alpha_{i}^{(1)}-\alpha_{i+1}^{(1)}, 0\right)\left(\beta_{i+\frac{1}{2}}^{(1,2)}\right)^{+} \mathbf{F}\left(\mathbf{U}_{i}^{(1)}, \mathbf{U}_{i+1}^{(2)}\right) \\
+ & \min \left(\alpha_{i}^{(1)}, \alpha_{i+1}^{(1)}\right) \mathbf{F}\left(\mathbf{U}_{i}^{(1)}, \mathbf{U}_{i+1}^{(1)}\right) \\
& -\max \left(\alpha_{i}^{(2)}-\alpha_{i+1}^{(2)}, 0\right)\left(\beta_{i+\frac{1}{2}}^{(2,1)}\right)^{-} \mathbf{F}\left(\mathbf{U}_{i}^{(2)}, \mathbf{U}_{i+1}^{(1)}\right), \\
\mathcal{E}\left(\mathbf{F}^{\ell a g} \frac{\partial X}{\partial x}\right)_{i, \text { bound }}= & \max \left(\alpha_{i}^{(1)}-\alpha_{i+1}^{(1)}, 0\right)\left(\beta_{i+\frac{1}{2}}^{(1,2)}\right)^{-} \mathbf{F}^{\ell a g}\left(\mathbf{U}_{i}^{(1)}, \mathbf{U}_{i+1}^{(2)}\right) \\
& -\max \left(\alpha_{i}^{(2)}-\alpha_{i+1}^{(2)}, 0\right)\left(\beta_{i+\frac{1}{2}}^{(2,1)}\right)^{-} \mathbf{F}^{\ell a g}\left(\mathbf{U}_{i}^{(2)}, \mathbf{U}_{i+1}^{(1)}\right) \\
& -\max \left(\alpha_{i-1}^{(1)}-\alpha_{i}^{(1)}, 0\right)\left(\beta_{i-\frac{1}{2}}^{(1,2)}\right)^{+} \mathbf{F}^{\ell a g}\left(\mathbf{U}_{i-1}^{(1)}, \mathbf{U}_{i}^{(2)}\right) \\
& +\max \left(\alpha_{i-1}^{(2)}-\alpha_{i}^{(2)}, 0\right)\left(\beta_{i-\frac{1}{2}}^{(2,1)}\right)^{+} \mathbf{F}^{\ell a g}\left(\mathbf{U}_{i-1}^{(2)}, \mathbf{U}_{i}^{(1)}\right),
\end{aligned}
$$

and

$$
\mathcal{E}\left(\mathbf{F}^{\ell a g} \frac{\partial X}{\partial x}\right)_{i, \text { relax }}=\lambda_{i}\left(\mathbf{F}^{\ell a g}\left(\mathbf{U}_{i}^{(2)}, \mathbf{U}_{i}^{(1)}\right)-\mathbf{F}^{\ell a g}\left(\mathbf{U}_{i}^{(1)}, \mathbf{U}_{i}^{(2)}\right)\right) .
$$

Setting $F=0$ and $U=1$ leads to the following numerical semidiscrete scheme for the volume fraction:

$$
\begin{aligned}
\frac{\mathrm{d} \alpha_{i}^{(1)}}{\mathrm{d} t}=\frac{1}{\Delta x} & {\left[\max \left(\alpha_{i}^{(1)}-\alpha_{i+1}^{(1)}, 0\right)\left(\beta_{i+\frac{1}{2}}^{(1,2)}\right)^{-} u^{\star}\left(\mathbf{U}_{i}^{(1)}, \mathbf{U}_{i+1}^{(2)}\right)\right.} \\
& -\max \left(\alpha_{i}^{(2)}-\alpha_{i+1}^{(2)}, 0\right)\left(\beta_{i+\frac{1}{2}}^{(2,1)}\right)^{-} u^{\star}\left(\mathbf{U}_{i}^{(2)}, \mathbf{U}_{i+1}^{(1)}\right) \\
& -\max \left(\alpha_{i-1}^{(1)}-\alpha_{i}^{(1)}, 0\right)\left(\beta_{i-\frac{1}{2}}^{(1,2)}\right)^{+} u^{\star}\left(\mathbf{U}_{i-1}^{(1)}, \mathbf{U}_{i}^{(2)}\right) \\
& \left.+\max \left(\alpha_{i-1}^{(2)}-\alpha_{i}^{(2)}, 0\right)\left(\beta_{i-\frac{1}{2}}^{(2,1)}\right)^{+} u^{\star}\left(\mathbf{U}_{i-1}^{(2)}, \mathbf{U}_{i}^{(1)}\right)\right] \\
& +\lambda_{i}\left\{u^{\star}\left(\mathbf{U}_{i}^{(2)}, \mathbf{U}_{i}^{(1)}\right)-u^{\star}\left(\mathbf{U}_{i}^{(1)}, \mathbf{U}_{i}^{(2)}\right)\right\} .
\end{aligned}
$$

In practical applications, the discretization is achieved via a splitting method. First, we integrate (with some abuse of notation ${ }^{3}$ )

$$
\frac{\left(W_{i}^{(1)}\right)^{n+1 / 2}-\left(W_{i}^{(1)}\right)^{n}}{\Delta t}+\frac{\mathcal{E}(X F)_{i+1 / 2}-\mathcal{E}(X F)_{i-1 / 2}}{\Delta x}=\frac{\mathcal{E}\left(F^{\ell a g} \frac{\partial X}{\partial x}\right)_{i, \text { bound }}}{\Delta x}
$$

\footnotetext{
${ }^{3}$ This is because (2.8) is not written as a difference of flux plus a relaxation term.
} 
and then a relaxation step

$$
\frac{\left(W_{i}^{(1)}\right)^{n+1}-\left(W_{i}^{(1)}\right)^{n+1 / 2}}{\Delta t}=\mathcal{E}\left(F^{\ell a g} \frac{\partial X}{\partial x}\right)_{i, \text { relax }} .
$$

The flux are computed at time $t_{n}$. The relaxation step is carried out implicitly.

2.3. Extension to second order. The scheme (2.9)-(2.10) makes use of several terms (the conservative terms, the boundary terms, and the relaxation terms) that do not play the same role in the derivation. The "conservative" terms are standard, so that any standard high order extension will do. The "relaxation" and "boundary" terms have the same status: they are the agglomeration of fluxes between different phases for which the high order procedure is not immediate.

In order to construct a high order extension of the scheme (2.9)-(2.10), the procedure described in [2] is considered:

1. We extrapolate the physical variables $\left(\alpha^{(\ell)}, u^{(\ell)}, P^{(\ell)}\right), \ell=1,2$, using the MUSCL method. Here, the minmod or van Leer-van Albada limiters are used.

2. We subdivide the cell $\mathcal{C}_{i}$ into $N$ regular subcells ( $N$ arbitrary) denoted by $\left[y_{l}, y_{l+1}\right](1 \leq l \leq N-1)$ and average in each subcell the reconstructed variables.

3. Each subcell is randomly subdivided as above.

4. Then we gather the contribution of each subsub-cell and let $N \rightarrow+\infty$.

In the following, $U_{i+1 / 2, l}^{(\ell)}$ (resp., $U_{i-1 / 2, r}^{(\ell)}$ ) represents the variable $U^{(\ell)}$ at the cell interface $x_{i+1 / 2}$ (resp., $x_{i-1 / 2}$ ) on the left (resp., right) after MUSCL extrapolation. If $f$ is any of the extrapolated variables, $\delta_{i} f$ represents its limited slope in $\mathcal{C}_{i}$. The jump in $\alpha^{(\ell)}$ at $y_{l+1 / 2}$ is $\Delta \alpha^{(\ell)}\left(y_{l+1 / 2}\right)$.

The nonconservative terms

$$
\mathcal{E}\left(F^{\ell a g} \frac{\partial X}{\partial x}\right)_{i}=\mathcal{E}\left(F^{\ell a g} \frac{\partial X}{\partial x}\right)_{i, \text { bound }}+\mathcal{E}\left(F^{\ell a g} \frac{\partial X}{\partial x}\right)_{i, \text { relax }}
$$

sum up to

$$
\begin{aligned}
& -\left(\beta_{i+1 / 2}^{(1,2)}\right)^{+} \mathcal{P}_{i+1 / 2}\left(\Sigma_{1}, \Sigma_{2}\right) \mathbf{F}^{\ell a g}\left(\mathbf{U}_{i+1 / 2, l}^{(1)}, \mathbf{U}_{i+1 / 2, r}^{(2)}\right) \\
& +\left(\beta_{i+1 / 2}^{(2,1)}\right)^{+} \mathcal{P}_{i+1 / 2}\left(\Sigma_{2}, \Sigma_{1}\right) \mathbf{F}^{\ell a g}\left(\mathbf{U}_{i+1 / 2, l}^{(2)}, \mathbf{U}_{i+1 / 2, r}^{(1)}\right) \\
& -\left(\beta_{i-1 / 2}^{(1,2)}\right)^{-} \mathcal{P}_{i-1 / 2}\left(\Sigma_{1}, \Sigma_{2}\right) \mathbf{F}^{\ell a g}\left(\mathbf{U}_{i-1 / 2, l}^{(1)}, \mathbf{U}_{i-1 / 2, r}^{(2)}\right) \\
& +\left(\beta_{i-1 / 2}^{(2,1)}\right)^{-} \mathcal{P}_{i-1 / 2}\left(\Sigma_{2}, \Sigma_{1}\right) \mathbf{F}^{\ell a g}\left(\mathbf{U}_{i-1 / 2, l}^{(2)}, \mathbf{U}_{i-1 / 2, r}^{(1)}\right) \\
& +\sum_{l=1}^{N-1} \max \left(0, \Delta \alpha^{(1)}\left(y_{l+1 / 2}\right)\right) \mathbf{F}^{\ell a g}\left(\mathbf{U}_{2}^{(2)}\left(y_{l+1 / 2}\right), \mathbf{U}_{1}^{(1)}\left(y_{l+1 / 2}\right)\right) \\
& \left.-\sum_{l=1}^{N-1} \max \left(0, \Delta \alpha^{(2)}\left(y_{l+1 / 2}\right)\right)\right) \mathbf{F}^{\ell a g}\left(\mathbf{U}_{2}^{(2)}\left(y_{l+1 / 2}\right), \mathbf{U}_{1}^{(1)}\left(y_{l+1 / 2}\right)\right) \\
& +\sum_{l=1}^{N-1} \lambda\left(y_{l+1 / 2}\right)\left(\mathbf{F}^{\ell a g}\left(\mathbf{U}_{2}^{(2)}\left(y_{l+1 / 2}\right), \mathbf{U}_{1}^{(1)}\left(y_{l+1 / 2}\right)\right)\right. \\
& \left.-\sum_{l=1}^{N-1} \mathbf{F}^{\ell a g}\left(\mathbf{U}_{1}^{(1)}\left(y_{l+1 / 2}\right), \mathbf{U}_{2}^{(2)}\left(y_{l+1 / 2}\right)\right)\right),
\end{aligned}
$$


where

$$
\Delta \alpha^{(1)}\left(y_{l+1 / 2}\right)=\left(\delta_{i} \alpha^{(1)}\right)\left(y_{l+1}-y_{l}\right)
$$

We do not need any additional $\beta$ terms because we account for all the internal cells in $] x_{i-1 / 2}, x_{i+1 / 2}[$.

The third and fourth terms of the above formula converge, when $N \rightarrow+\infty$, to

$$
\begin{gathered}
\left(\int_{x_{i-1 / 2}}^{x_{i+1 / 2}} \mathbf{F}^{\ell a g}\left(\mathbf{U}^{(2)}(y), \mathbf{U}^{(1)}(y)\right) d y\right) \max \left(0, \delta_{i} \alpha^{(1)}\right) \\
-\left(\int_{x_{i-1 / 2}}^{x_{i+1 / 2}} \mathbf{F}^{\ell a g}\left(\mathbf{U}^{(1)}(y), \mathbf{U}^{(2)}(y)\right) d y\right) \max \left(0, \delta_{i} \alpha^{(2)}\right) .
\end{gathered}
$$

A similar formula is obtained for the phase $\Sigma_{2}$. The integrals in (2.12) are evaluated via the midpoint formula (since second order accuracy is sought).

Similarly, the relaxation terms corresponding to a linear reconstruction of the data can be approximated, thanks to the same interpretation in terms of Riemann sums and to the midpoint rule by

$$
\lambda_{i}\left(\mathbf{F}^{\ell a g}\left(\mathbf{U}_{i}^{(2)}, \mathbf{U}_{i}^{(1)}\right)-\mathbf{F}^{\ell a g}\left(\mathbf{U}_{i}^{(1)}, \mathbf{U}_{i}^{(2)}\right)\right) .
$$

We finally obtain a second order approximation of the nonconservative terms by

$$
\begin{aligned}
\Delta x \mathcal{E}\left(\mathbf{F}^{\ell a g} \frac{\partial X}{\partial x}\right)= & -\left(\beta_{i+1 / 2}^{(1,2)}\right)^{+} \mathcal{P}_{i+1 / 2}\left(\Sigma_{1}, \Sigma_{2}\right) \mathbf{F}^{\ell a g}\left(\mathbf{U}_{i+1 / 2, l}^{(1)}, \mathbf{U}_{i+1 / 2, r}^{(2)}\right) \\
& +\left(\beta_{i+1 / 2}^{(2,1)}\right)^{+} \mathcal{P}_{i+1 / 2}\left(\Sigma_{2}, \Sigma_{1}\right) \mathbf{F}^{\ell a g}\left(\mathbf{U}_{i+1 / 2, l}^{(2)}, \mathbf{U}_{i+1 / 2, r}^{(1)}\right) \\
& -\left(\beta_{i-1 / 2}^{(1,2)}\right)^{-} \mathcal{P}_{i-1 / 2}\left(\Sigma_{1}, \Sigma_{2}\right) \mathbf{F}^{\ell a g}\left(\mathbf{U}_{i-1 / 2, l}^{(1)}, \mathbf{U}_{i-1 / 2, r}^{(2)}\right) \\
& +\left(\beta_{i-1 / 2}^{(2,1)}\right)^{-} \mathcal{P}_{i-1 / 2}\left(\Sigma_{2}, \Sigma_{1}\right) \mathbf{F}^{\ell a g}\left(\mathbf{U}_{i-1 / 2, l}^{(2)}, \mathbf{U}_{i-1 / 2, r}^{(1)}\right) \\
& +\lambda_{i}\left(\mathbf{F}^{\ell a g}\left(\mathbf{U}_{i}^{(2)}, \mathbf{U}_{i}^{(1)}\right)-\mathbf{F}^{\ell a g}\left(\mathbf{U}_{i}^{(1)}, \mathbf{U}_{i}^{(2)}\right)\right) \\
& +\max \left(0, \delta \alpha_{i}^{(1)}\right) \mathbf{F}^{\ell a g}\left(\mathbf{U}_{i}^{(2)}, \mathbf{U}_{i}^{(1)}\right) \\
& -\max \left(0, \delta \alpha_{i}^{(2)}\right) \mathbf{F}^{\ell a g}\left(\mathbf{U}_{i}^{(1)}, \mathbf{U}_{i}^{(2)}\right)
\end{aligned}
$$

where $\delta \alpha_{i}^{(1)}=\alpha_{i+1 / 2, l}^{(1)}-\alpha_{i-1 / 2, r}^{(1)}$ and $\delta \alpha_{i}^{(2)}=\alpha_{i+1 / 2, l}^{(2)}-\alpha_{i-1 / 2, r}^{(2)}$ are the limited slope of $\alpha^{(1)}$ and $\alpha^{(2)}$ in the cell $C_{i}$.

Hence, the second order extension of the scheme can be geometrically interpreted by adding an additional interface inside the cell $\mathcal{C}_{i}$; this is the meaning of last two terms of (2.14).

2.4. Extension to other solvers. In (2.7), the numerical flux $F$ that we use at the microscopic level is obtained thanks to an approximate Riemann solver for which it is possible to define a contact speed. The contact speed between the left state $U_{L}$ and the right state $U_{R}$ is denoted by $\sigma\left(U_{L}, U_{R}\right)$. We also denote by $U_{L R}^{ \pm}$ the left and right states surrounding the approximate contact discontinuity, similarly 


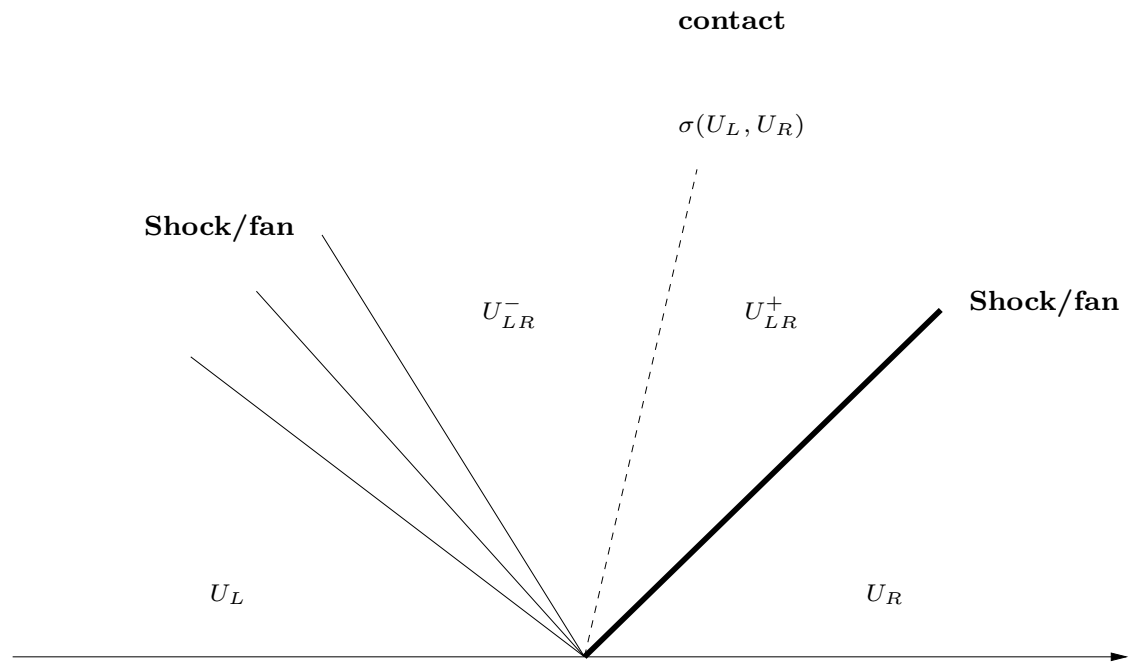

FIG. 2. Structure of the Riemann problem.

as in Figure 2 for the Godunov solver. This permits us to define the Lagrangian flux $F^{\ell a g}\left(U_{L}, U_{R}\right)=F\left(U_{L R}^{+}\right)-\sigma\left(U_{L}, U_{R}\right) U_{L R}^{+}=F\left(U_{L R}^{-}\right)-\sigma\left(U_{L}, U_{R}\right) U_{L R}^{-}$. Last, we define $\beta_{i+1 / 2}^{(p, q)}=\operatorname{sign}\left(\sigma\left(U_{i}^{(p)}, U_{i+1}^{(q)}\right)\right)$.

All the calculations have been performed for the Godunov scheme but can be extended to more general fluxes. The key ingredients of the derivation are, besides the randomization, average procedures, and estimation of the various coefficients, the use of a Riemann solver for which it is possible to define a contact discontinuity speed $\sigma(U, V)$. This property is needed because we must define a Lagrangian flux, $F^{\ell a g}(U, V)=F(U, V)-\sigma(U, V) U^{\star}$. This Lagrangian flux has to be consistent, as well as the base flux $F(U, V)$.

Even more, the choice of the base flux $F$ and the Lagrangian flux $F^{\ell a g}$ may be independent: we do not really need a relation of the type $F^{\ell a g}=F^{*}-\sigma U^{\star}$; see [2] for more details.

3. Asymptotic model for the numerical scheme. To get the numerical scheme for the five equations model (1.2), we propose following a similar technique as in [8], where the continuous model was derived via an asymptotic expansion of (1.1). The very difference is that we will do it at a discrete level. If we set $\varepsilon_{i}=\frac{1}{\lambda_{i}}$, the discrete scheme for the seven equations model can be formally rewritten as

$$
\frac{\partial W}{\partial t}+\frac{G}{\Delta x}=\frac{R(W)}{\varepsilon_{i}}
$$

and we assume that $\varepsilon_{i}$ is large.

The first step to get the asymptotic scheme is to determine the equilibrium set

$$
\{W \text { such that } R(W)=0\} \text {. }
$$

From the previous section, this set is defined as

$$
\mathcal{V}=\left\{W=\left(\alpha^{(1)}, \alpha^{(1)} \mathbf{U}^{(1)}, \alpha^{(2)}, \alpha^{(2)} \mathbf{U}^{(2)}\right) \text { such that } F^{\ell a g}\left(W_{1}, W_{2}\right)=F^{\ell a g}\left(W_{2}, W_{1}\right)\right\} .
$$


Then the limit model is obtained by developing the variables around the equilibrium set. We assume that $\mathcal{V}$ can be parametrized by a mapping $M: \mathbf{u} \mapsto M(\mathbf{u})$ (we prove this fact later) and that $R(W)$ has an expansion of the form

$$
R(W)=R(M(u))+\varepsilon R^{\prime}(M(u)) V+o(\varepsilon) .
$$

Thus (3.1) becomes

$$
\mathrm{d} M_{\mathbf{u}} \frac{\partial u}{\partial t}+\frac{G}{\Delta x}=R^{\prime}(M(\mathbf{u})) V+o(1) .
$$

To get the reduced model, it remains to multiply by the projection $P$ onto the kernel of $R^{\prime}(M(\mathbf{u}))$ in the direction of $\mathrm{d} M_{\mathbf{u}}$. This gives the model

$$
P \mathrm{~d} M_{\mathbf{u}} \frac{\partial u}{\partial t}+P \frac{G}{\Delta x}=0 .
$$

The aim of this section is to show that for several classical solvers, $P$ does not depend on the solver. We have to show two properties:

1. $\mathcal{V}$ is equal to

$$
\left\{\left(\alpha^{(1)}, \alpha^{(1)} \mathbf{U}^{(1)}, \alpha^{(2)}, \alpha^{(2)} \mathbf{U}^{(2)}\right) \text { such that } u_{1}=u_{2} \text { and } P_{1}=P_{2}\right\}
$$

as in the continuous case.

2. The Jacobian matrix of $F^{\ell a g}\left(\mathbf{U}^{(1)}, \mathbf{U}^{(2)}\right)-F^{\ell a g}\left(\mathbf{U}^{(2)}, \mathbf{U}^{(1)}\right)$ in primitive variables is

$$
\mathrm{D} R(M(u))=\left(\begin{array}{cccccccc}
0 & 0 & 0 & -\lambda^{\prime} & 0 & 0 & 0 & \lambda^{\prime} \\
0 & 0 & 0 & 0 & 0 & 0 & 0 & 0 \\
0 & 0 & \mu^{\prime} & 0 & 0 & 0 & -\mu^{\prime} & 0 \\
0 & 0 & \mu^{\prime} u & \lambda^{\prime} P & 0 & 0 & -\mu^{\prime} u & -\lambda^{\prime} P \\
0 & 0 & 0 & \lambda^{\prime} & 0 & 0 & 0 & -\lambda^{\prime} \\
0 & 0 & 0 & 0 & 0 & 0 & 0 & 0 \\
0 & 0 & -\mu^{\prime} & 0 & 0 & 0 & \mu^{\prime} & 0 \\
0 & 0 & -\mu^{\prime} u & -\lambda^{\prime} P & 0 & 0 & \mu^{\prime} u & \lambda^{\prime} P
\end{array}\right)
$$

as in the continuous case.

In the following, we denote by a subscript 12 (resp., 21) the intermediate states obtained by solving an elementary Riemann problem before averaging with left and right states given by the phase $\Sigma_{1}$ (resp., $\Sigma_{2}$ ) on the left and $\Sigma_{2}$ (resp., $\Sigma_{1}$ ) on the right.

3.1. Case of the acoustic solver. Given two states, left and right, described by the physical variables, $\left(\rho_{L}, u_{L}, P_{L}\right)$ for the left state and $\left(\rho_{R}, u_{R}, P_{R}\right)$ for the right state, the Lagrangian flux for the acoustic solver is defined as $\left(0, P^{\star}, P^{\star} u^{\star}\right)$ with the pressure and the velocity of the intermediate $\operatorname{state}(\star)$ given by (see, e.g., [9])

$$
\begin{aligned}
P^{\star} & =\frac{Z_{R} P_{L}+Z_{L} P_{R}+Z_{L} Z_{R}\left(u_{L}-u_{R}\right)}{Z_{R}+Z_{L}}, \\
u^{\star} & =\frac{Z_{R} u_{R}+Z_{L} u_{L}+P_{L}-P_{R}}{Z_{R}+Z_{L}} .
\end{aligned}
$$

In (3.6), $Z_{L, R}=\rho_{L, R} a_{L, R}$ is the acoustic impedance. Therefore, using the above mentioned conventions, and using the fact that within the cell we consider the left/right 
states are defined from the conservative variables $U^{(1)}$ for $\Sigma_{1}$ and $U^{(2)}$ for $\Sigma_{2}$, we have

$$
\begin{array}{ll}
P_{12}^{\star}=\frac{Z_{1} P_{1}+Z_{2} P_{2}+Z_{1} Z_{2}\left(u_{2}-u_{1}\right)}{Z_{2}+Z_{1}}, & P_{21}^{\star}=\frac{Z_{1} P_{1}+Z_{2} P_{2}+Z_{1} Z_{2}\left(u_{1}-u_{2}\right)}{Z_{1}+Z_{2}}, \\
u_{12}^{\star}=\frac{Z_{1} u_{1}+Z_{2} u_{2}+P_{2}-P_{1}}{Z_{1}+Z_{2}}, & u_{21}^{\star}=\frac{Z_{1} u_{1}+Z_{2} u_{2}+P_{1}-P_{2}}{Z_{1}+Z_{2}}
\end{array}
$$

so that

$$
\begin{aligned}
& u_{12}^{\star}-u_{21}^{\star}=\frac{2}{Z_{1}+Z_{2}}\left(P_{1}-P_{2}\right), \\
& P_{12}^{\star}-P_{21}^{\star}=\frac{2 Z_{1} Z_{2}}{Z_{1}+Z_{2}}\left(u_{1}-u_{2}\right) .
\end{aligned}
$$

We immediately see that if $u_{12}^{\star}-u_{21}^{\star}=0$ and $P_{12}^{\star}-P_{21}^{\star}=0$, we have $u_{1}=u_{2}$ and $P_{1}=P_{2}$.

Let us evaluate now the Jacobian matrix of $\mathbf{F}^{\ell a g}\left(U^{(1)}, U^{(2)}\right)-\mathbf{F}^{\ell a g}\left(U^{(2)}, U^{(1)}\right)$ with respect to the primitive variable on $\mathcal{V}$. We have

$$
\mathbf{F}^{\ell a g}\left(U^{(1)}, U^{(2)}\right)-\mathbf{F}^{\ell a g}\left(U^{(2)}, U^{(1)}\right)=\left(0, P_{12}^{\star}-P_{21}^{\star}, P_{12}^{\star} u_{12}^{\star}-P_{21}^{\star} u_{21}^{\star}\right),
$$

and we have to evaluate the partial derivative of this vector with respect to $v=\rho, u$, and $P$. Using the fact that the considered state lies in $\mathcal{V}$, a straightforward calculation gives (with $u=u_{1}=u_{2}$ and $P=P_{1}=P_{2}$ )

$$
\begin{gathered}
\frac{\partial\left(P_{12}^{\star}-P_{21}^{\star}\right)}{\partial v}=\frac{2}{Z_{1}+Z_{2}}\left(\frac{\partial P_{1}}{\partial v}-\frac{\partial P_{2}}{\partial v}\right) \\
\frac{\partial\left(P_{12}^{\star} u_{12}^{\star}-P_{21}^{\star} u_{21}^{\star}\right)}{\partial v}=\frac{2 Z_{1} Z_{2}}{Z_{1}+Z_{2}} u\left(\frac{\partial P_{1}}{\partial v}-\frac{\partial P_{2}}{\partial v}\right)+\frac{2}{Z_{1}+Z_{2}} P\left(\frac{\partial u_{1}}{\partial v}-\frac{\partial u_{2}}{\partial v}\right)
\end{gathered}
$$

so that we get (3.5) with

$$
\lambda=\frac{2}{Z_{1}+Z_{2}} \quad \text { and } \quad \mu=\frac{2 Z_{1} Z_{2}}{Z_{1}+Z_{2}} .
$$

3.2. Case of the exact solver. For the exact solver, the Lagrangian flux can be written as $\left(0, P^{\star}, P^{\star} u^{\star}\right)$, where $P^{\star}$ and $u^{\star}$ are the intermediate velocity and pressure when the Riemann problem is solved exactly. Equation (3.4) can be rewritten as

$$
\left(0, P_{12}^{\star}, P_{12}^{\star} u_{12}^{\star}\right)=\left(0, P_{21}^{\star}, P_{21}^{\star} u_{21}^{\star}\right)
$$

so that

$$
u^{\star}=u_{21}^{\star}=u_{12}^{\star} \quad \text { and } \quad P^{\star}=P_{21}^{\star}=P_{12}^{\star} .
$$

Thus in the $(u, P)$ variables, the point $\left(u^{\star}, P^{\star}\right)$ lies on the 1 -wave curve coming from the state $\left(u_{1}, P_{1}\right)$ and on the 3 -wave curve coming from the state $\left(u_{1}, P_{1}\right)$. Therefore $u^{\star}=u_{1}$ and $P^{\star}=P_{1}$, because the 1 -wave curve is decreasing and the 3 -wave curve is increasing: this is a consequence of the convexity of the equation of state [7, page 89]. The same argument holds for the state 2 , so that $u^{\star}=u_{2}$ and $P^{\star}=P_{2}$ so that $u_{1}=u_{2}$ and $P_{1}=P_{2}$.

Now we estimate the Jacobian matrix. Indeed, we find exactly the same Jacobian matrix as for the acoustic solver because the relations (3.6) are obtained by a linearization in the $(u, P)$ variables of the true Riemann invariants at $\left(u=u_{1}=\right.$ $\left.u_{2}, P=P_{1}=P_{2}\right)$. 
3.3. Case of the HLLC solver. The HLLC solver is based on the choice of two wave velocities $S^{L}$ and $S^{R}$. The intermediate states are calculated by writing the Rankine-Hugoniot relations across these waves; see [11, page 293] for more details. With this solver, the intermediates states are given by

$$
\mathbf{U}_{K}^{\star}=\rho_{K} \frac{S^{K}-u_{K}}{S^{K}-u^{\star}}\left(\begin{array}{c}
1 \\
u_{K}^{\star}+\left(u^{\star}-u_{K}\right)\left(u^{\star}+\frac{P_{K}}{\rho_{K}\left(S^{K}-u_{K}\right)}\right)
\end{array}\right)
$$

with $K=L$ or $R$. The Lagrangian flux is then

$$
\mathbf{F}^{\ell a g}=\left(\begin{array}{c}
0 \\
\rho_{K}\left(u^{\star}-u_{K}\right)\left(S^{L}-u_{L}\right)+P_{L} \\
\left(\rho_{K}\left(u^{\star}-u_{K}\right)\left(S^{L}-u_{L}\right)+P_{L}\right) u^{\star}
\end{array}\right) .
$$

Thus $\mathbf{F}_{12}^{\ell a g}$ and $\mathbf{F}_{21}^{\ell a g}$ are given by the following expressions:

$$
\begin{aligned}
\mathbf{F}_{12}^{\ell a g} & =\left(\begin{array}{c}
0 \\
\rho_{1}\left(u_{12}^{\star}-u_{1}\right)\left(S_{12}^{L}-u_{1}\right)+P_{1} \\
\left(\rho_{1}\left(u_{12}^{\star}-u_{1}\right)\left(S_{12}^{L}-u_{1}\right)+P_{1}\right) u_{12}^{\star}
\end{array}\right) \\
& =\left(\begin{array}{c}
0 \\
\rho_{2}\left(u_{12}^{\star}-u_{2}\right)\left(S_{12}^{R}-u_{2}\right)+P_{2} \\
\left(\rho_{2}\left(u_{12}^{\star}-u_{2}\right)\left(S_{12}^{R}-u_{2}\right)+P_{2}\right) u_{12}^{\star}
\end{array}\right), \\
\mathbf{F}_{21}^{\ell a g} & =\left(\begin{array}{c}
\rho_{1}\left(u_{21}^{\star}-u_{1}\right)\left(S_{21}^{R}-u_{1}\right)+P_{1} \\
\left(\rho_{1}\left(u_{21}^{\star}-u_{1}\right)\left(S_{21}^{R}-u_{1}\right)+P_{1}\right) u_{21}^{\star}
\end{array}\right) \\
& =\left(\begin{array}{c} 
\\
0 \\
\rho_{2}\left(u_{21}^{\star}-u_{2}\right)\left(S_{21}^{L}-u_{2}\right)+P_{2} \\
\left(\rho_{2}\left(u_{21}^{\star}-u_{2}\right)\left(S_{21}^{L}-u_{2}\right)+P_{2}\right) u_{21}^{\star}
\end{array}\right) .
\end{aligned}
$$

The intermediate velocity is given by

$$
u^{\star}=\frac{P_{R}-P_{L}+\rho_{L} u_{L}\left(S^{L}-u_{L}\right)-\rho_{R} u_{R}\left(S^{R}-u_{R}\right)}{\rho_{L}\left(S^{L}-u_{L}\right)-\rho_{R}\left(S^{R}-u_{R}\right)} .
$$

For more convenience, we denote by

$$
\left\{\begin{array}{l}
Z^{L}=\rho_{L}\left(u_{L}-S^{L}\right) \\
Z^{R}=\rho_{R}\left(S^{R}-u_{R}\right) .
\end{array}\right.
$$

Then (3.8) becomes

$$
u^{\star}=\frac{P_{L}-P_{R}+Z^{R} u_{R}+Z^{L} u_{L}}{Z^{R}+Z^{L}} .
$$

Here we have used the same notation convention as in section 3.1 for the velocity and pressure. The subscripts 1 and 2 in $Z$ are intended to highlight the fact that the 
definition of $Z$ depends not only on the left and right state through $\rho$ and $u$ but also on the ordering of the waves through the speed $S$. For example, $Z_{1}^{12}$ is evaluated with $U^{(1)}$ on the left and $U^{(2)}$ on the right and corresponds to the leftmost wave $S$. Thus we have

$$
\begin{aligned}
& u_{12}^{\star}-u_{21}^{\star}= \frac{P_{2}-P_{1}+Z_{1}^{21} u_{1}+Z_{2}^{21} u_{2}}{Z_{1}^{21}+Z_{2}^{21}}-\frac{P_{1}-P_{2}+Z_{1}^{12} u_{1}+Z_{2}^{12} u_{2}}{Z_{1}^{12}+Z_{2}^{12}} \\
&= \frac{Z_{1}^{21}+Z_{2}^{21}+Z_{1}^{12}+Z_{2}^{12}}{\left(Z_{1}^{21}+Z_{2}^{21}\right)\left(Z_{1}^{12}+Z_{2}^{12}\right)}\left(P_{2}-P_{1}\right)+\frac{Z_{1}^{21} Z_{2}^{12}-Z_{2}^{21} Z_{1}^{12}}{\left(Z_{1}^{21}+Z_{2}^{21}\right)\left(Z_{1}^{12}+Z_{2}^{12}\right)} u_{1} \\
&-\frac{Z_{1}^{21} Z_{2}^{12}-Z_{2}^{21} Z_{1}^{12}}{\left(Z_{1}^{21}+Z_{2}^{21}\right)\left(Z_{1}^{12}+Z_{2}^{12}\right)} u_{2} \\
&=A\left(u_{1}-u_{2}\right)+B\left(P_{2}-P_{1}\right)
\end{aligned}
$$

with

$$
\begin{aligned}
& A=\frac{Z_{1}^{21} Z_{2}^{12}-Z_{2}^{21} Z_{1}^{12}}{\left(Z_{1}^{21}+Z_{2}^{21}\right)\left(Z_{1}^{12}+Z_{2}^{12}\right)}, \\
& B=\frac{Z_{1}^{21}+Z_{2}^{21}+Z_{1}^{12}+Z_{2}^{12}}{\left(Z_{1}^{21}+Z_{2}^{21}\right)\left(Z_{1}^{12}+Z_{2}^{12}\right)} .
\end{aligned}
$$

Let us examine the difference between the second component of (3.7a) and (3.7c), i.e., $P_{12}^{\star}-P_{21}^{\star}$. We need to evaluate $u^{\star}-u_{L}$ and $u^{\star}-u_{R}$. From (3.8), we have

$$
\begin{aligned}
& u^{\star}-u_{R}=\frac{P_{L}-P_{R}+Z^{L}\left(u_{L}-u_{R}\right)}{Z^{R}+Z^{L}}, \\
& u^{\star}-u_{L}=\frac{P_{L}-P_{R}+Z^{R}\left(u_{R}-u_{L}\right)}{Z^{R}+Z^{L}} .
\end{aligned}
$$

This leads to

$$
\begin{aligned}
P_{12}^{\star}-P_{21}^{\star}= & Z_{2}^{12}\left(u_{12}^{\star}-u_{2}\right)+Z_{2}^{21}\left(u_{21}^{\star}-u_{2}\right) \\
= & Z_{2}^{12} \frac{P_{1}-P_{2}+Z_{1}^{12}\left(u_{1}-u_{2}\right)}{Z_{1}^{12}+Z_{2}^{12}}+Z_{2}^{21} \frac{P_{2}-P_{1}+Z_{1}^{21}\left(u_{1}-u_{2}\right)}{Z_{1}^{21}+Z_{2}^{21}} \\
= & \frac{Z_{1}^{21} Z_{2}^{12}-Z_{2}^{21} Z_{1}^{12}}{\left(Z_{1}^{21}+Z_{2}^{21}\right)\left(Z_{1}^{12}+Z_{2}^{12}\right)}\left(P_{1}-P_{2}\right) \\
& \quad+\left(\frac{Z_{2}^{12} Z_{1}^{12}}{Z_{1}^{12}+Z_{2}^{12}}+\frac{Z_{2}^{21} Z_{1}^{21}}{Z_{1}^{21}+Z_{2}^{21}}\right)\left(u_{1}-u_{2}\right) \\
& =C\left(u_{1}-u_{2}\right)-A\left(P_{2}-P_{1}\right)
\end{aligned}
$$

with

$$
C=\frac{Z_{2}^{12} Z_{1}^{12}}{Z_{1}^{12}+Z_{2}^{12}}+\frac{Z_{2}^{21} Z_{1}^{21}}{Z_{1}^{21}+Z_{2}^{21}}
$$

Clearly, the system

$$
\left\{\begin{array}{l}
u_{12}^{\star}-u_{21}^{\star}=0, \\
P_{12}^{\star}-P_{21}^{\star}=0
\end{array}\right.
$$


is equivalent to

$$
\left\{\begin{array}{l}
A\left(u_{1}-u_{2}\right)+B\left(P_{2}-P_{1}\right)=0 \\
C\left(u_{1}-u_{2}\right)-A\left(P_{2}-P_{1}\right)=0
\end{array}\right.
$$

which has a determinant $-A^{2}-B C$.

For the system to be invertible, it is sufficient that $B$ and $C$ be strictly positive. This is true in particular if all the $Z$ are strictly positive. We show later in this section and for several choices of speeds $S_{L, R}$ that $Z>0$, and thus the system is invertible.

Assume the system is invertible: the equilibrium set is (3.4).

It remains to evaluate the Jacobian matrix along the equilibrium set $\mathcal{V}$. This is done as in section 3.1. For example, if we compute the derivative of $P_{12}^{\star}-P_{21}^{\star}$ with respect to $v=\rho, u$, or $P$, we have

$$
\frac{\partial\left(P_{12}^{\star}-P_{21}^{\star}\right)}{\partial v}=\frac{\partial A}{\partial v}\left(P_{1}-P_{2}\right)+\frac{\partial B}{\partial v}\left(u_{1}-u_{2}\right)+A \frac{\partial\left(P_{1}-P_{2}\right)}{\partial v}+B \frac{\partial\left(u_{1}-u_{2}\right)}{\partial v} .
$$

If the variables belong to $\mathcal{V}$, we have

$$
\frac{\partial\left(P_{12}^{\star}-P_{21}^{\star}\right)}{\partial v}=A \frac{\partial\left(P_{1}-P_{2}\right)}{\partial v}+B \frac{\partial\left(u_{1}-u_{2}\right)}{\partial v} .
$$

Therefore, if $A=0$ in the equilibrium set, we get the result for $P_{12}^{\star}-P_{21}^{\star}$. The same result holds for $u_{12}^{\star}-u_{21}^{\star}$. Thus, the only thing left is to show that $A$ is zero along (3.4).

The remainder of this section is devoted to showing, for several classical choices of speeds $S[11]$, that

1. $Z>0$,

2. $A$ defined in $(3.9 \mathrm{~b})$ satisfies $A=0$ along $\mathcal{V}$.

3.3.1. Case where $S^{L}=u_{L}-a_{L}$ and $S^{R}=u_{R}+a_{R}$. We have

$$
\begin{aligned}
& Z_{1}^{12}=\rho_{1} a_{1}, \\
& Z_{2}^{12}=\rho_{2} a_{2}, \\
& Z_{1}^{21}=\rho_{1} a_{1}, \\
& Z_{2}^{21}=\rho_{2} a_{2},
\end{aligned}
$$

and we see immediately that the $Z$ 's are all strictly positive and that, directly from (3.9b), $A=0$ along $\mathcal{V}$.

3.3.2. Case where $S^{L}=\min \left(u_{L}-a_{L}, u_{R}-a_{R}\right)$ and $S^{R}=\max$ $\left(\boldsymbol{u}_{\boldsymbol{R}}+\boldsymbol{a}_{\boldsymbol{R}}, \boldsymbol{u}_{\boldsymbol{L}}+\boldsymbol{a}_{\boldsymbol{L}}\right)$. In this case, it is obvious that the $Z$ 's are strictly positive so that the equilibrium set is (3.4). Along this equilibrium set, we denote by $\bar{a}$ the maximum sound speed and $u$ the velocity of each fluid. We have

$$
\begin{aligned}
& Z_{1}^{12}=\rho_{1} \bar{a} \\
& Z_{2}^{12}=\rho_{2} \bar{a} \\
& Z_{1}^{21}=\rho_{1} \bar{a} \\
& Z_{2}^{21}=\rho_{2} \bar{a}
\end{aligned}
$$

therefore, $A=0$ along the equilibrium set. 
3.3.3. Case where $S^{L}=\tilde{u}-\tilde{a}$ and $S^{R}=\tilde{u}+\tilde{a}$, where $\tilde{x}$ is the Roe average $^{4}$ of $\boldsymbol{x}$. The equation which gives the intermediate velocity no longer holds because we are not sure that the denominator is nonzero. Nevertheless, we still can use the equations (3.7). The equality of the second and third component of (3.7a) and $(3.7 \mathrm{~b})$ gives immediately

$$
u_{12}^{\star}=u_{21}^{\star}
$$

and we denote by $u^{\star}$ this velocity. Moreover, by abstracting the second component of $(3.7 \mathrm{c})$ and the second component of $(3.7 \mathrm{a})$, and by doing the same algebra with $(3.7 \mathrm{~b})$ and $(3.7 \mathrm{~d})$, we get

$$
\left\{\begin{array}{l}
\left(u^{\star}-u_{2}\right)\left(S_{12}^{L}-S_{21}^{R}\right)=0 \\
\left(u^{\star}-u_{1}\right)\left(S_{21}^{L}-S_{12}^{R}\right)=0
\end{array}\right.
$$

Here the wave velocities are symmetric in $u_{1}$ and $u_{2}$, so that $S_{12}^{L}=S_{21}^{L}=S^{L}$ and $S_{21}^{R}=S_{12}^{R}=S^{R}$. We have $S^{L}-S^{R}=-2 \tilde{a} \neq 0$, which leads to

$$
u_{1}=u_{2}=u^{\star} \text {. }
$$

Last, the equality of the second component of $(3.7 \mathrm{a})$ and $(3.7 \mathrm{~b})$ provides $P_{1}=P_{2}$. Thus, the equilibrium set is once more (3.4). Along the equilibrium set, we have

$$
\begin{aligned}
& Z_{1}^{12}=\rho_{1} \tilde{a}, \\
& Z_{2}^{12}=\rho_{2} \tilde{a}, \\
& Z_{1}^{21}=\rho_{1} \tilde{a}, \\
& Z_{2}^{21}=\rho_{2} \tilde{a},
\end{aligned}
$$

where $a$ is the Roe average of $a_{1}$ and $a_{2}$, so that $Z$ 's are strictly positive, and we can use the formula (3.9a) for $A$ which gives immediately $A=0$ on $\mathcal{V}$.

3.3.4. Case of pressure-velocity-based wave speed estimates. A different approach, that works for perfect gases only, consists in first estimating the pressure $P^{\star}$ and $u^{\star}$ and then derivating the estimates of $S^{L}$ and $S^{R}$. If we suppose that we have estimated $P^{\star}$ and $u^{\star}$, then we choose the following wave speeds:

$$
S^{L}=u_{L}-a_{L} q_{L}, \quad S^{R}=u_{R}+a_{R} q_{R},
$$

where

$$
q_{K}=\left\{\begin{array}{lll}
1 & \text { if } \quad P^{\star} \leq P_{K}, \\
\left(1+\frac{\gamma+1}{2 \gamma}\left(\frac{P^{\star}}{P_{K}}-1\right)\right)^{\frac{1}{2}} & \text { if } \quad P^{\star}>P_{K} .
\end{array}\right.
$$

Then we find

$$
\begin{aligned}
& Z_{1}^{12}=\rho_{1} a_{1} q_{L}^{12}, \\
& Z_{2}^{12}=\rho_{2} a_{2} q_{R}^{12}, \\
& Z_{1}^{21}=\rho_{1} a_{1} q_{R}^{21}, \\
& Z_{2}^{21}=\rho_{2} a_{2} q_{L}^{21} .
\end{aligned}
$$

\footnotetext{
${ }^{4} \tilde{x}:=\frac{\sqrt{\rho_{L}} x_{L}+\sqrt{\rho_{R}} x_{R}}{\sqrt{\rho_{L}}+\sqrt{\rho_{R}}}$.
} 
These $Z$ 's are all strictly nonnegative, so that the equilibrium set is (3.4). On the equilibrium set, $P_{12}^{\star}=P_{21}^{\star}$ so that with (3.12) we have

$$
q_{L}^{12}=q_{R}^{21} \quad \text { and } \quad q_{R}^{12}=q_{L}^{21},
$$

and $A$ is null on the equilibrium set.

3.4. Case of the relaxation solver of [3]. We approximate the solutions of the Euler equations with those of the following relaxation system:

$$
\left\{\begin{array}{l}
\frac{\partial \rho}{\partial t}+\frac{\partial(\rho u)}{\partial x}=0 \\
\frac{\partial(\rho u)}{\partial t}+\frac{\partial\left(\rho u^{2}+\pi\right)}{\partial x}=0 \\
\frac{\partial(\rho E)}{\partial t}+\frac{\partial((\rho E+\pi) u)}{\partial x}=0 \\
\frac{\partial \pi}{\partial t}+u \frac{\partial \pi}{\partial x}+\frac{a^{2}}{\rho} \frac{\partial u}{\partial x}=\frac{P-\pi}{\eta}
\end{array}\right.
$$

where $a$ is a parameter chosen to ensure the system is dissipative when the relaxation time $\eta \rightarrow \infty(a>\max (\rho c))$. The solution of the Riemann problem for (3.13) can easily be computed because all the fields are linearly degenerate. In particular, we find the following formulas for the intermediate pressure and velocity:

$$
\begin{aligned}
\pi^{\star} & =\frac{\pi_{R}+\pi_{L}}{2}+\frac{a}{2}\left(u_{L}-u_{R}\right), \\
u^{\star} & =\frac{\pi_{L}-\pi_{R}}{2 a}+\frac{u_{L}+u_{R}}{2} .
\end{aligned}
$$

Thus we immediately get

$$
\begin{aligned}
& P_{12}^{\star}-P_{21}^{\star}=\frac{a_{12}+a_{21}}{2}\left(u_{1}-u_{2}\right), \\
& u_{12}^{\star}-u_{21}^{\star}=\frac{1}{2}\left(\frac{1}{a_{12}}+\frac{1}{a_{21}}\right)\left(P_{1}-P_{2}\right) .
\end{aligned}
$$

As in the case of the acoustic solver, we see immediately that the equilibrium set is (3.4). Last, the study of the Jacobian matrix with the same method as before shows that (3.5) is true with

$$
\lambda=\frac{1}{2}\left(\frac{1}{a_{12}}+\frac{1}{a_{21}}\right) \quad \text { and } \quad \mu=\frac{a_{12}+a_{21}}{2} .
$$

4. Derivation of the numerical scheme for the five equations model. In the previous section, we have shown that, for several classical solvers, the equilibrium variety is the same as the one in the continuous case, namely

$$
\mathcal{V}=\left\{u_{1}=u_{2} \quad \text { and } \quad P_{1}=P_{2}\right\}
$$


Therefore, $\mathcal{V}$ is of dimension 6 and can be parametrized by the mapping $M$ :

$$
M:\left(\begin{array}{c}
\alpha_{1} \\
\rho_{1} \\
u \\
P \\
\alpha_{2} \\
\rho_{2}
\end{array}\right) \mapsto\left(\begin{array}{c}
\alpha_{1} \\
\rho_{1} \\
u \\
P \\
\alpha_{2} \\
\rho_{2} \\
u \\
P
\end{array}\right) .
$$

Since this parametrization is naturally written in primitive variables and not in conservative variables, the first thing to do is to provide the form of the numerical scheme for the seven equations model in primitive variables, which is equivalent to the form in conserved variables.

The next step is to compute the projector $P$ onto the kernel of $R^{\prime}(M(\mathbf{u}))$ in the direction of $\mathrm{d} M_{\mathbf{u}}$ and then to evaluate (3.3).

4.1. Transformation into primitive form. In order to simplify the algebra, we rewrite (3.1) as

$$
\begin{aligned}
& \frac{\partial \alpha_{i}^{(k)}}{\partial t}=V F_{i}^{(k)} \\
& \frac{\partial\left(\alpha_{i}^{(k)} \rho_{i}^{(k)}\right)}{\partial t}=M_{a} F_{i}^{(k)} \\
& \frac{\partial\left(\alpha_{i}^{(k)} \rho_{i}^{(k)} u_{i}^{(k)}\right)}{\partial t}=M_{o} F_{i}^{(k)} \\
& \frac{\partial\left(\alpha_{i}^{(k)} \rho_{i}^{(k)} E_{i}^{(k)}\right)}{\partial t}=E F_{i}^{(k)}
\end{aligned}
$$

Since

$$
\alpha_{i}^{(k)} \rho_{i}^{(k)} \frac{\partial u_{i}^{(k)}}{\partial t}=M_{o} F_{i}^{(k)}-u_{i}^{(k)} M_{a} F_{i}^{(k)}
$$

we have

$$
\begin{aligned}
\frac{1}{2} \frac{\partial\left(\alpha_{i}^{(k)} \rho_{i}^{(k)} u_{i}^{(k)^{2}}\right)}{\partial t} & =\frac{u_{i}^{(k)}}{2} \frac{\partial\left(\alpha_{i}^{(k)} \rho_{i}^{(k)} u_{i}^{(k)}\right)}{\partial t}+\frac{\alpha_{i}^{(k)} \rho_{i}^{(k)} u_{i}^{(k)}}{2} \frac{\partial u_{i}^{(k)}}{\partial t} \\
& =\frac{1}{2} u_{i}^{(k)} M_{o} F_{i}^{(k)}+\frac{1}{2} u_{i}^{(k)}\left(M_{o} F_{i}^{(k)}-u_{i}^{(k)} M_{a} F_{i}^{(k)}\right) \\
& =\frac{1}{2} u_{i}^{(k)} M_{o} F_{i}^{(k)}+\frac{1}{2} u_{i}^{(k)} M_{o} F_{i}^{(k)}-\frac{u_{i}^{(k)}}{2} M_{a} F_{i}^{(k)} \\
& =u_{i}^{(k)} M_{o} F_{i}^{(k)}-\frac{\left(u_{i}^{(k)}\right)^{2}}{2} M_{a} F_{i}^{(k)}
\end{aligned}
$$

Similarly,

$$
E F_{i}^{(k)}=\frac{\partial\left(\alpha_{i}^{(k)} \rho_{i}^{(k)} E_{i}^{(k)}\right)}{\partial t}=\frac{\partial\left(\alpha_{i}^{(k)} \rho_{i}^{(k)} \varepsilon_{i}^{(k)}\right)}{\partial t}+\frac{1}{2} \frac{\partial\left(\alpha_{i}^{(k)} \rho_{i}^{(k)}\left(u_{i}^{(k)}\right)^{2}\right)}{\partial t}
$$


and

$$
\frac{\partial\left(\alpha_{i}^{(k)} \rho_{i}^{(k)} \varepsilon_{i}^{(k)}\right)}{\partial t}=E F_{i}^{(k)}-u_{i}^{(k)} M_{o} F_{i}^{(k)}+\frac{\left(u_{i}^{(k)}\right)^{2}}{2} M_{a} F_{i}^{(k)} .
$$

We develop the energy equation to get an equation on the pressure

$$
\begin{aligned}
\frac{\partial\left(\alpha_{i}^{(k)} \rho_{i}^{(k)} \varepsilon_{i}^{(k)}\right)}{\partial t} & =\varepsilon_{i}^{(k)} \frac{\partial\left(\alpha_{i}^{(k)} \rho_{i}^{(k)}\right)}{\partial t}+\alpha_{i}^{(k)} \rho_{i}^{(k)} \frac{\partial \varepsilon_{i}^{(k)}}{\partial t} \\
& =\varepsilon_{i}^{(k)} M_{a} F_{i}^{(k)}+\alpha_{i}^{(k)} \rho_{i}^{(k)} \beta_{i}^{(k)} \frac{\partial P_{i}^{(k)}}{\partial t}+\alpha_{i}^{(k)} \rho_{i}^{(k)} \kappa_{i}^{(k)} \frac{\partial \rho_{i}^{(k)}}{\partial t}
\end{aligned}
$$

where

$$
\beta_{i}^{(k)}=\left(\frac{\partial \varepsilon_{i}^{(k)}}{\partial P_{i}^{(k)}}\right)_{\rho_{i}^{(k)}} \quad \text { and } \quad \kappa_{i}^{(k)}=\left(\frac{\partial \varepsilon_{i}^{(k)}}{\partial \rho_{i}^{(k)}}\right)_{P_{i}^{(k)}} .
$$

When we combine the mass and the volume fraction equations, we obtain

$$
\alpha_{i}^{(k)} \frac{\partial \rho_{i}^{(k)}}{\partial t}=M_{a} F_{i}^{(k)}-\rho_{i}^{(k)} V F_{i}^{(k)} .
$$

Then we have

$$
\begin{aligned}
\alpha_{i}^{(k)} \rho_{i}^{(k)} \beta_{i}^{(k)} \frac{\partial P_{i}^{(k)}}{\partial t}=E F_{i}^{(k)}- & u_{i}^{(k)} M_{o} F_{i}^{(k)}+\frac{u_{i}^{(k)^{2}}}{2} M_{a} F_{i}^{(k)}-\varepsilon_{i}^{(k)} M_{a} F_{i}^{(k)} \\
& -\alpha_{i}^{(k)} \rho_{i}^{(k)} \kappa_{i}^{(k)} \frac{\partial \rho_{i}^{(k)}}{\partial t} \\
=E F_{i}^{(k)}- & u_{i}^{(k)} M_{o} F_{i}^{(k)}+\frac{u_{i}^{(k)}}{2} M_{a} F_{i}^{(k)}-\varepsilon_{i}^{(k)} M_{a} F_{i}^{(k)} \\
& -\rho_{i}^{(k)} \kappa_{i}^{(k)}\left(M_{a} F_{i}^{(k)}-\rho_{i}^{(k)} V F_{i}^{(k)}\right),
\end{aligned}
$$

that is,

$$
\begin{aligned}
\frac{\partial P_{i}^{(k)}}{\partial t} & =\frac{E F_{i}^{(k)}}{\alpha_{i}^{(k)} \rho_{i}^{(k)} \beta_{i}^{(k)}}-\frac{u_{i}^{(k)} M_{o} F_{i}^{(k)}}{\alpha_{i}^{(k)} \rho_{i}^{(k)} \beta_{i}^{(k)}} \\
& +\frac{\left(\frac{u_{i}^{(k)}}{2}-\varepsilon_{i}^{(k)}-\rho_{i}^{(k)} \kappa_{i}^{(k)}\right)}{\alpha_{i}^{(k)} \rho_{i}^{(k)} \beta_{i}^{(k)}} M_{a} F_{i}^{(k)}+\frac{\rho_{i}^{(k)}{ }^{2} \kappa_{i}^{(k)} V F_{i}^{(k)}}{\alpha_{i}^{(k)} \rho_{i}^{(k)} \beta_{i}^{(k)}} .
\end{aligned}
$$

The scheme (2.7) is equivalent to (4.4), (4.3), and (4.5).

4.2. The projection. Formally, the seven equations numerical scheme in primitive variables is

$$
\frac{\partial \mathbf{U}^{\prime}}{\partial t}+\frac{G^{\prime}}{\Delta x}=T \frac{R\left(\mathbf{U}^{\prime}\right)}{\varepsilon_{j}},
$$


where $T$ is the linear transformation between primitive and conservative variables

$$
T=\left(\begin{array}{cc}
T_{1} & 0 \\
0 & T_{2}
\end{array}\right)
$$

with

$$
T_{i}=\left(\begin{array}{cccc}
1 & 0 & 0 & 0 \\
-\frac{\rho_{i}}{\alpha_{i}} & \frac{1}{\alpha_{i}} & 0 & 0 \\
0 & -\frac{u_{i}}{\alpha_{i}} & \frac{1}{\alpha_{i} \rho_{i}} & 0 \\
\frac{\kappa_{i} \rho_{i}^{2}}{\alpha_{i} \rho_{i} \beta_{i}} & \frac{\frac{u_{i}^{2}}{2}-\varepsilon_{i}-\rho_{i} \kappa_{i}}{\alpha_{i} \rho_{i} \beta_{i}} & -\frac{u}{\alpha_{i} \rho_{i} \beta_{i}} & \frac{1}{\alpha_{i} \rho_{i} \beta_{i}}
\end{array}\right)
$$

The range of $\mathrm{D} R(M(u))$ is spanned by

$$
\mathbf{V}_{1}=(1,0,0,-P,-1,0,0, P) \quad \text { and } \quad \mathbf{V}_{2}=(0,0,1, u, 0,0,-1,-u),
$$

and we want to project in the direction of ${ }^{5}$

$$
T \mathbf{V}_{1}=\left(\begin{array}{c}
0 \\
0 \\
\alpha_{2} \rho_{2} \\
0 \\
0 \\
-\alpha_{1} \rho_{1} \\
0 \\
\end{array}\right) \quad \text { and } \quad T \mathbf{V}_{2}=\left(\begin{array}{c}
1 \\
-\frac{\rho_{1}}{\alpha_{1}} \\
0 \\
-\frac{\rho_{1} a_{1}^{2}}{\alpha_{1}} \\
\frac{\rho_{2}}{\alpha_{2}} \\
0 \\
\frac{\rho_{2} a_{2}^{2}}{\alpha_{2}}
\end{array}\right) .
$$

A tedious but straightforward calculation shows that the matrix of the projector is

$$
\Pi=\left(\begin{array}{ccccccc}
1 & 0 & 0 & \frac{\alpha_{1} \alpha_{2}}{d} & 0 & 0 & -\frac{\alpha_{1} \alpha_{2}}{d} \\
0 & 1 & 0 & -\frac{\rho_{1} \alpha_{2}}{d} & 0 & 0 & \frac{\rho_{1} \alpha_{2}}{d} \\
0 & 0 & \frac{\rho_{1} \alpha_{1}}{\alpha_{1} \rho_{1}+\alpha_{2} \rho_{2}} & 0 & 0 & \frac{\rho_{2} \alpha_{2}}{\alpha_{1} \rho_{1}+\alpha_{2} \rho_{2}} & 0 \\
0 & 0 & 0 & \frac{\alpha_{1} \rho_{2} a_{2}^{2}}{d} & 0 & 0 & \frac{\alpha_{2} \rho_{1} a_{1}^{2}}{d} \\
0 & 0 & 0 & \frac{\alpha_{1} \rho_{2}}{d} & 1 & 0 & -\frac{\alpha \rho_{2}}{d}
\end{array}\right)
$$

with

$$
d=\alpha_{1} \rho_{2}\left(a_{2}\right)^{2}+\alpha_{2} \rho_{1}\left(a_{1}\right)^{2}
$$

By putting back the system into the reduced conservative variables

$$
\left(\alpha_{i}, \alpha_{i} \rho_{i}, \rho u, \rho E\right)
$$

\footnotetext{
${ }^{5}$ Note that $\frac{P_{k}-\kappa_{k} \rho_{k}^{2}}{\beta_{k} \rho_{k}^{2}}=a_{k}^{2}$.
} 
the evaluation of (3.3) provides the following scheme for the five equations model:

$$
\begin{aligned}
\frac{\partial \alpha_{2}}{\partial t}=F V_{2}+\frac{\alpha_{1} \alpha_{2}}{\alpha_{2} \rho_{1} a_{1}^{2}+\alpha_{1} \rho_{2} a_{2}^{2}} & \left(\frac{S E_{2}}{\alpha_{2} \rho_{2} \beta_{2}}-\frac{u_{2} S U_{2}}{\alpha_{2} \rho_{2} \beta_{2}}+\frac{\left(\frac{u_{2}^{2}}{2}-\varepsilon_{2}-\rho_{2} \kappa_{2}\right)}{\alpha_{2} \rho_{2} \beta_{2}} M_{2}\right. \\
& +\frac{\rho_{2}^{2} \kappa_{2} F V_{2}}{\alpha_{2} \rho_{2} \beta_{2}}-\frac{S E_{1}}{\alpha_{1} \rho_{1} \beta_{1}}+\frac{u_{1} S U_{1}}{\alpha_{1} \rho_{1} \beta_{1}}-\frac{\left(\frac{u_{1}^{2}}{2}-\varepsilon_{1}-\rho_{1} \kappa_{1}\right)}{\alpha_{1} \rho_{1} \beta_{1}} M_{1} \\
& \left.-\frac{\rho_{1}^{2} \kappa_{1} F V_{1}}{\alpha_{1} \rho_{1} \beta_{1}}\right) \\
\frac{\partial\left(\alpha_{k} \rho_{k}\right)}{\partial t}=M_{k}, & \\
\frac{\partial(\rho u)}{\partial t}=S U_{1}+S U_{2}, &
\end{aligned}
$$

where we have used the notations of (4.2).

We conclude this section by noting that this applies to the first order as well as the second order scheme.

5. Numerical results. In the numerical tests, all the fluids were described by the Stiffened-gas equation of state (EOS):

$$
\varepsilon(P, \rho)=\frac{P+\gamma P^{\infty}}{(\gamma-1) \rho},
$$

where $\gamma$ and $P^{\infty}$ are constants that depend on the phase under consideration. In this paper, the fluids used in the numerical tests are the water and the air, which have coefficients $\gamma$ and $P^{\infty}$ given in Tables 3 and 6 . In some cases, one side of the tube will be filled with pure fluids, in which case the volume fraction of the fluid that is not here will be initialized with $\alpha_{\epsilon}=10^{-8}$. All examples are run with a CFL number of 0.6. The CFL number is computed on the largest wave speed in the flow.

The test cases are chosen so that one can see the influence of the div $\mathbf{u}$ terms and are ordered by increasing difficulty. The last case is a comparison with experimental data.

We have chosen to use different solvers in the experiments. Several considerations have motivated our choices: the best possible accuracy and the most possible robustness. In interface-like problems, we need to initialize one of the fluids with a very small volume fraction, typically of the order of $10^{-8}$. In that case, the solver "sees" the fluid as vacuum and might be very sensitive to any numerical error, especially in the case of complex and stiff wave systems. Because of that, some solvers are not suited to some cases. In the following, we have chosen to use the acoustic solver in the tests of section 5.2 (in fact all the solvers are working fine on this case) and the relaxation solver on the other ones. This choice seems to be the best compromise. 
TABLE 3

EOS coefficients for water and air.

\begin{tabular}{|l|l|}
\hline \multicolumn{1}{|c|}{ Air } & \multicolumn{1}{c|}{ Water } \\
\hline$\gamma=1.4$ & $\gamma=4.4$ \\
$P^{\infty}=0 \mathrm{~Pa}$ & $P^{\infty}=6.10^{8} \mathrm{~Pa}$ \\
\hline
\end{tabular}

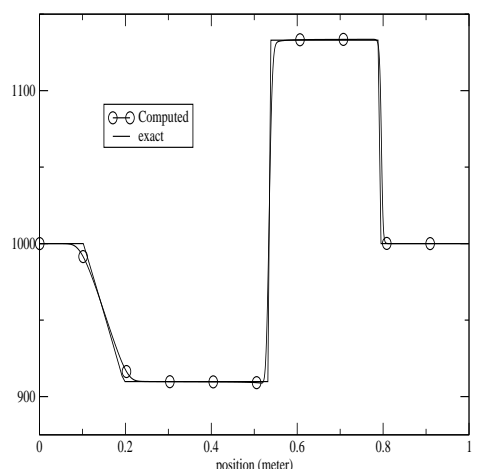

Density

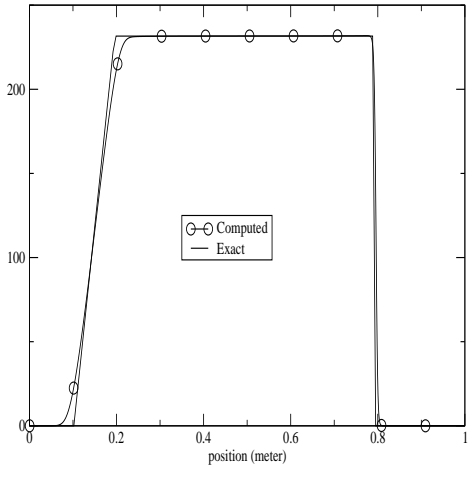

Velocity

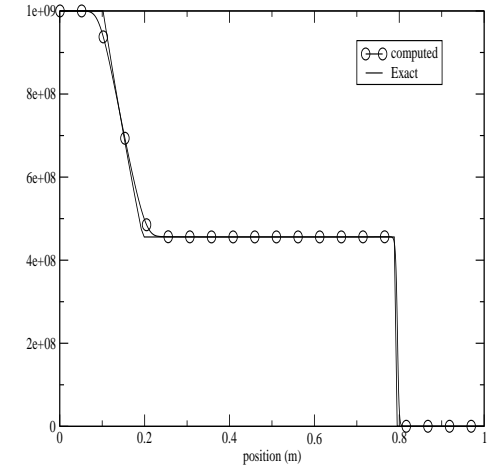

Pressure

FIG. 3. Single fluid problem: approximated solution (circles) and exact solution (solid).

5.1. Single fluid test. In that case, the shock tube is filled with water only. At time $t=0$, the left part of the tube is at rest, with a pressure of $10^{9} \mathrm{~Pa}$, and the right side is at rest too but with a pressure of $10^{5} \mathrm{~Pa}$. The separation between both sides is at $x=0.5 \mathrm{~m}$, and the profiles are shown at $t=150 \mu \mathrm{s}$. The approximated solution is computed with a 1000 point grid mesh and is compared with the analytical solution in Figure 3. The second order scheme has been run. The exact solution and the numerical one are in good agreement.

5.2. Pure interface advection. A one meter long tube is filled on the right with air and on the left with water. The pressure is uniform, equal to $10^{5} \mathrm{~Pa}$, and the velocity is uniform too, equal to $1000 \mathrm{~m} \cdot \mathrm{s}^{-1}$. At time $t=0$, the discontinuity is localized at $x=0.5 \mathrm{~m}$. The exact solution consists in the advection of mass at $1000 \mathrm{~m} . \mathrm{s}^{-1}$, and the pressure and the velocity remain uniform. The results are shown at time $t=200 \mu$ s when the exact discontinuity is located at $x=0.7 \mathrm{~m}$. The solution is computed with a 1000 cell mesh and is shown in Figure 4. The results show that 
the velocity and the pressure remain uniform, and the density and volume of fluids are simply advected, as expected.

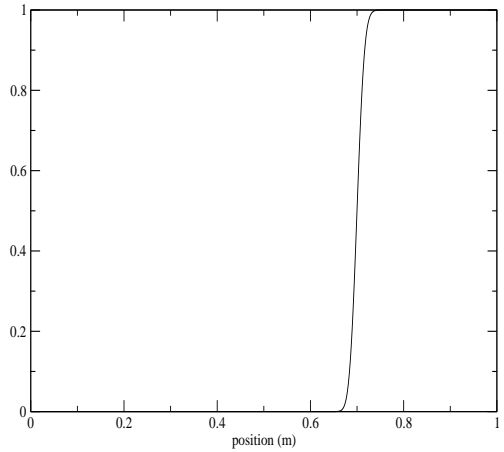

Volume of Fluids

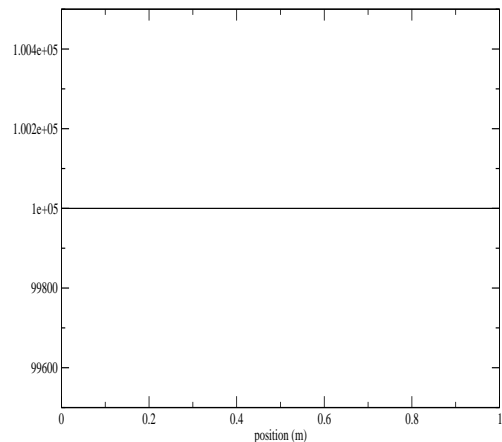

Pressure

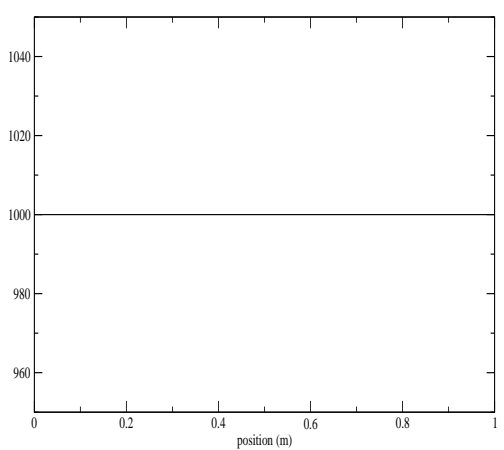

Velocity

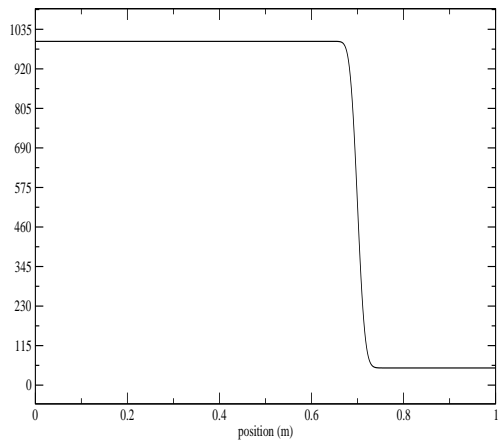

Density

FIG. 4. Pure fluid advection: the interface is diffused and centered around $x=0.7 \mathrm{~m}$. The pressure and velocity are left unchanged.

5.3. Liquid-gas shock tube. Here we study the evolution of a tube filled on the left with high-pressure water $\left(10^{9} \mathrm{~Pa}\right)$ and on the right with atmospheric-pressure air $\left(10^{5} \mathrm{~Pa}\right)$. The discontinuity is initially at $x=0,7 \mathrm{~m}$. The details of the initial conditions are given in Table 4. The computed solutions of the present scheme and of [2] are compared in Figure 5. 
TABLE 4

Details of the conditions for the liquid-gas shock tube of section 5.3.

\begin{tabular}{|l|l|}
\hline \multicolumn{2}{|c|}{ Fluid 1: air } \\
\hline $\begin{array}{l}\alpha_{1}=\alpha_{\epsilon} \\
\rho_{1}=50 \mathrm{~kg} . \mathrm{m}^{-3}\end{array}$ & $\begin{array}{l}\alpha_{1}=1-\alpha_{\epsilon} \\
\rho_{1}=50 \mathrm{~kg} \cdot \mathrm{m}^{-3}\end{array}$ \\
\hline \multicolumn{2}{|c|}{ Fluid 2: water } \\
\hline$\alpha_{2}=1-\alpha_{\epsilon}$ & $\alpha_{2}=\alpha_{\epsilon}$ \\
$\rho_{2}=1000 \mathrm{~kg} \cdot \mathrm{m}^{-3}$ & $\rho_{2}=1000 \mathrm{~kg} \cdot \mathrm{m}^{-3}$ \\
\hline \multicolumn{2}{|c|}{ Global variables } \\
\hline$P=10^{9} \mathrm{~Pa}$ & $P=10^{5} \mathrm{~Pa}$ \\
$u=0 \mathrm{~m} \cdot \mathrm{s}^{-1}$ & $u=0 \mathrm{~m} . \mathrm{s}^{-1}$ \\
\hline
\end{tabular}

Note that these solutions are computed with the second order scheme. In Figure 6, we display the first and second order accurate solutions for this problem. Clearly, the second order results are much more accurate than the first order ones, as expected.

5.4. Two phase flow problem. In that case, the left and the right part of the shock tube are filled with water and air at the same volume fraction of 0.5. The pressure is of $10^{9} \mathrm{~Pa}$ on the left and of $10^{5} \mathrm{~Pa}$ on the right. The discontinuity is initially at $x=0.5 \mathrm{~m}$. The details of the initial conditions are given in Table 5 . The computed solutions of the present scheme and of [2] are compared in Figure 7. The results are in good agreement, but we note some discrepancies. The main difference between the present schemes and that of [2], as it can be seen in Figure 7, consists in the density levels between the contact and the shock wave. The location of the discontinuities as well as the extreme points of the fan coincide exactly up to numerical errors of course. Note, however, that the average densities almost coincide between the contact and the shock and do coincide elsewhere. The pressure and velocity plots superimpose up to numerical errors. In our opinion, the fact that the discontinuities move at the same speed is a good indication that we are computing the same system: our experience indicates that in the case of nonconservative systems, as here, a small modification in the approximation of nonconservative terms implies a large modification of the numerical wave speeds. These are certainly a consequence of the different techniques in time integration: in the original scheme, the relaxation terms are integrated by a splitting technique, but one has to remember that the relaxation terms originate from fluxes. On the contrary, in the present technique, no splitting technique is used. The asymptotic expansion we use has some features of a direct integration of the relaxation terms inside the solver, which is probably better in principle. These algorithmic differences seem here to have a large impact on the volume fraction, but we have no clear explanation why. In Figure 8, we have represented a zoom of the average density between the contact discontinuity and the shock wave for the first and second order versions of the present scheme and the original one. We clearly see the (small) differences. The most interesting phenomenon is that, first, the two first order solutions are very close and, second, the second order solution for the present scheme is much closer from the first order ones than is the second order version of the original scheme. From the algorithmic point of view, the original second order scheme uses a predictor corrector method, and the predicted quantities are projected onto the equilibrium manifold after the predictor and the corrector steps. In the present scheme, this is translated into the choice of the states that are needed in the computation of the projector $\Pi$ defined in (4.7). In the second order version of the present scheme, $\Pi$ is evaluated after each substep. Hence, there is no reason why the two sets of results should coincide exactly. It appears, however, that the 


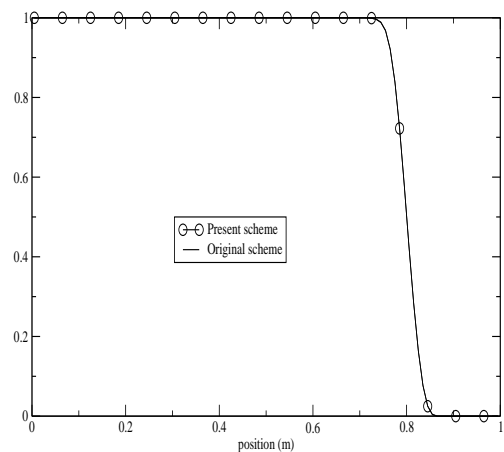

Liquid volume fraction

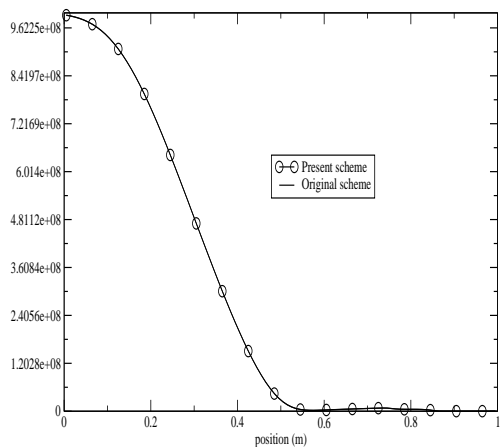

Pressure

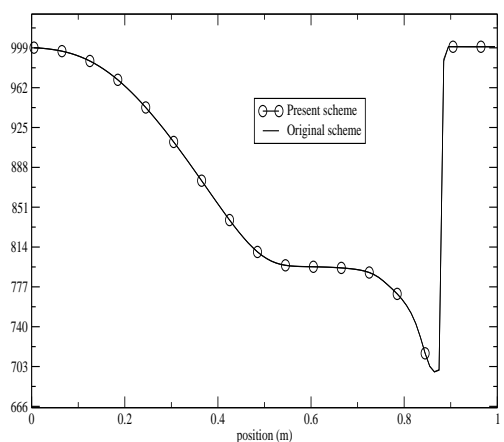

Liquid density

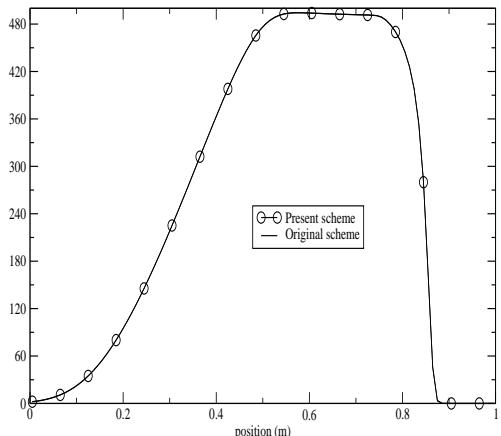

Velocity

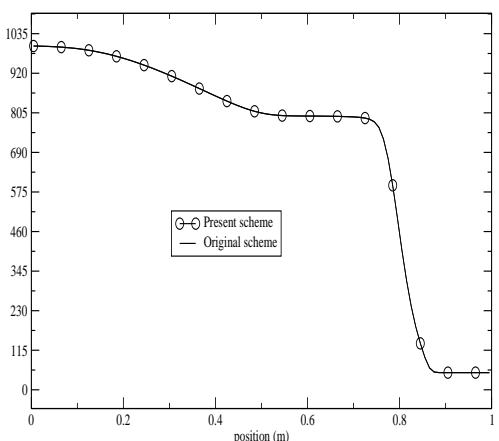

Average density

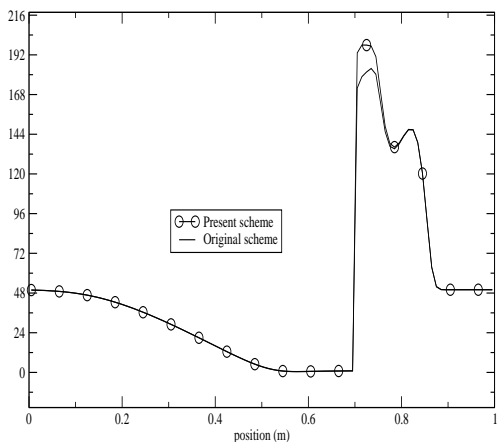

Gas density

FIG. 5. Liquid-gas shock tube: Comparison between the solutions obtained by the original scheme of [2] (circles) and the present scheme (solid). Both solutions differ only on the densities, in zones where they have few meaning because the corresponding volume fractions are nearly zero.

present technique, based on an asymptotic expansion, is a more stable technique than a simple fractional step method. The lack of exact solution does not enable more 

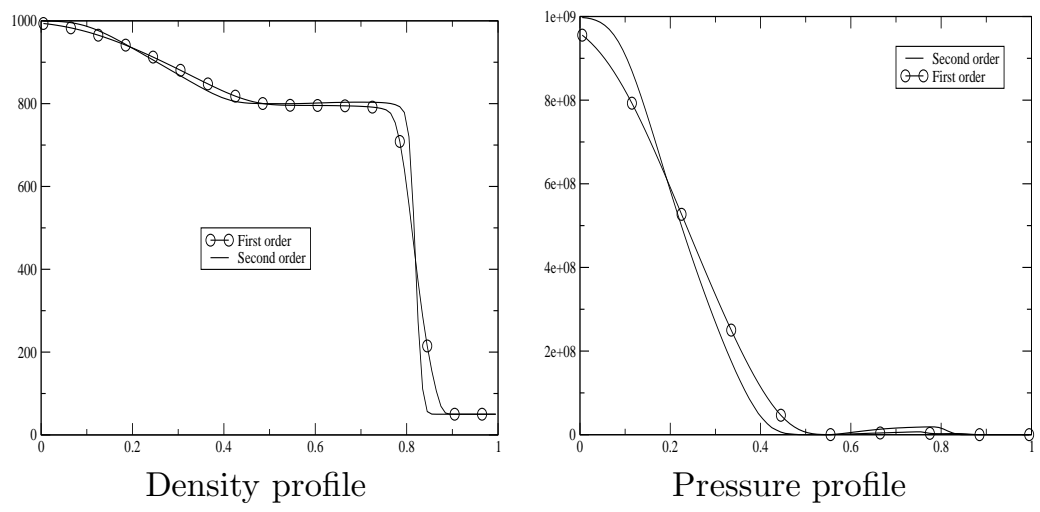

FIG. 6. Comparison between the first and second order accurate schemes.

TABLE 5

Details of the conditions for the two phase flow problem of section 5.4.

\begin{tabular}{|l|l|}
\hline \multicolumn{2}{|c|}{ Fluid 1: air } \\
\hline $\begin{array}{l}\alpha_{1}=0.5 \\
\rho_{1}=50 \mathrm{~kg} \cdot \mathrm{m}^{-3}\end{array}$ & $\begin{array}{l}\alpha_{1}=0.5 \\
\rho_{1}=50 \mathrm{~kg} \cdot \mathrm{m}^{-3}\end{array}$ \\
\hline \multicolumn{2}{|c|}{ Fluid 2: water } \\
\hline$\alpha_{2}=0.5$ & $\alpha_{2}=0.5$ \\
$\rho_{2}=1000 \mathrm{~kg} \cdot \mathrm{m}^{-3}$ & $\rho_{2}=1000 \mathrm{~kg} \cdot \mathrm{m}^{-3}$ \\
\hline \multicolumn{2}{|c|}{ Global variables } \\
\hline$P=10^{9} \mathrm{~Pa}$ & $P=10^{5} \mathrm{~Pa}$ \\
$u=0 \mathrm{~m} . \mathrm{s}^{-1}$ & $u=0 \mathrm{~m} . \mathrm{s}^{-1}$ \\
\hline
\end{tabular}

accurate comments.

5.5. Comparison with experiment. We make a simulation on an alloy made of epoxy and spinel for which experimental data exist. The tube is impacted with a piston, so that a shock propagates. The experimental setup is sketched in Figure 9. There exists a linear relation between the shock speed $u_{c}$ and the impact velocity $u_{i}$ : $u_{c}=a_{0}+s u_{i}$. Epoxy and spinel are described by the stiffened-gas EOS with the coefficient of Table 6 .

Initially, both of the solids are at atmospheric pressure, and their densities are $\rho_{1}=1185 \mathrm{~kg} \cdot \mathrm{m}^{-3}$ and $\rho_{2}=3622 \mathrm{~kg} \cdot \mathrm{m}^{-3}$. Their respective volume fractions are $\alpha_{1}=$ 0.595 and $\alpha_{2}=0,405$. We measure the different variables in $x=0.1 \mathrm{~m}$ and in $x=0.6 \mathrm{~m}$. Thanks to these measures (shown in Figure 10) we can compute the shock speed. In Figure 11, we compare our results with the experimental measures taken from [6], those of [8], and those obtained by the original scheme and the present scheme.

We see that our results are very close to those of the original scheme and in good agreement with the experimental results. In particular, we see that there is an excellent agreement between the results of the seven equation model with instantaneous relaxation and the present scheme. The agreement is much better than with the Murrone and Guillard scheme for which the div $\mathbf{u}$ terms are discretized independently because

- our results are closer from the experimental ones, 

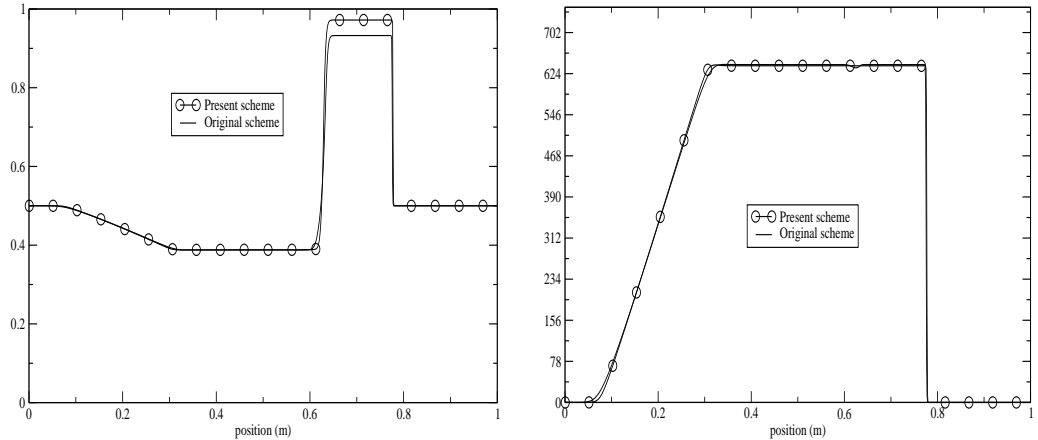

Liquid volume fraction

Velocity
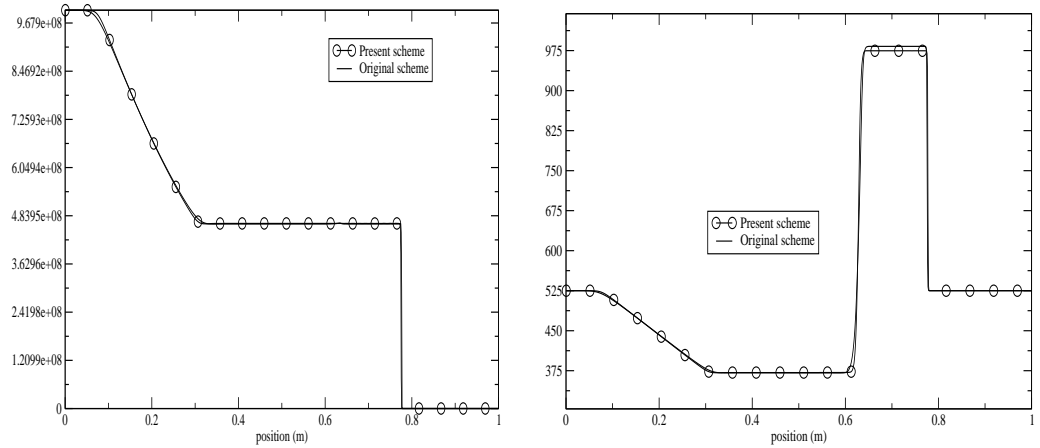

Pressure

Average density

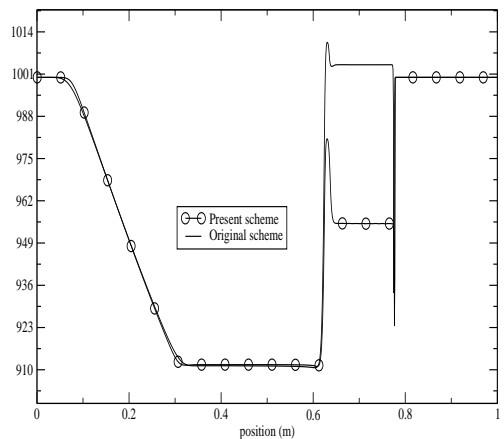

Liquid density

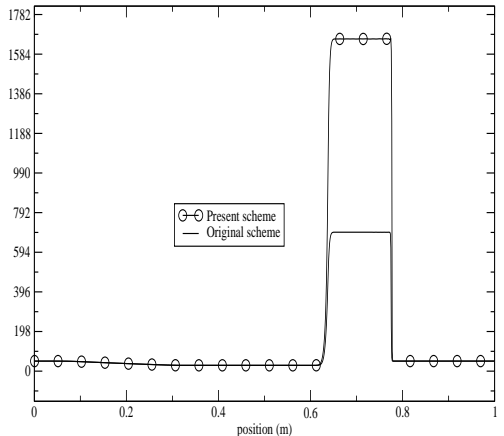

Gas density

FIG. 7. Two phase flow problem: the present scheme (circles) is compared with the original scheme of [2] (solid lines).

- there is very little difference between the results with the complete seven equation model and the reduced five equations model discretized with our technique. 


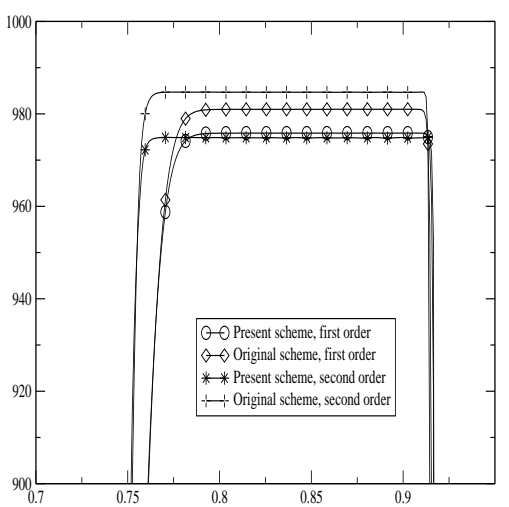

FIG. 8. Comparison of the first and second order version of the original scheme and the present one for the averaged density. Zoom between the contact discontinuity and the shock wave for the data of Table 5 .

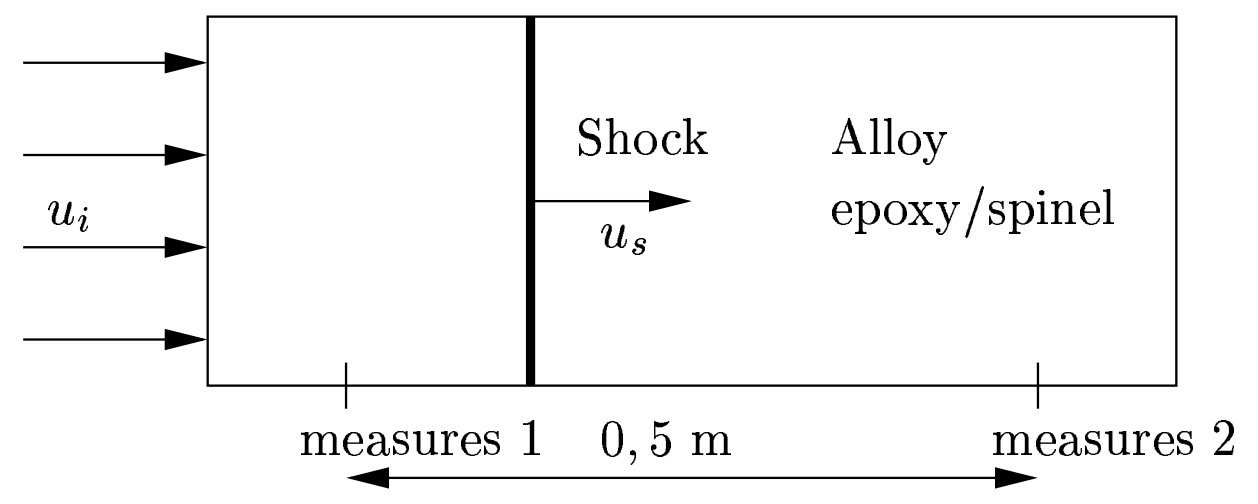

FIG. 9. Experimental setup.

TABLE 6

EOS coefficients for epoxy and spinel.

\begin{tabular}{|l|l|}
\hline \multicolumn{1}{|c|}{ Epoxy } & \multicolumn{1}{|c|}{ Spinel } \\
\hline$\gamma=2.94$ & $\gamma=1.62$ \\
$P^{\infty}=3.2 \times 10^{9} \mathrm{PaPa}$ & $P^{\infty}=141 \times 10^{8} \mathrm{~Pa}$ \\
\hline
\end{tabular}

6. Conclusions. We have presented a numerical scheme able to compute compressible multiphase schemes that relies on a multiscale description of the flow. Three types of terms can be identified in the scheme in addition to the temporal terms. One is a conservative term, the second one plays the role of a nonconservative term that is related to interfacial quantities, and the last one is a relaxation term that is associated with acoustic phenomena. The key feature of the scheme is that it is locally conservative, contrarily to many other schemes devoted to compressible multiphase problems. The scheme is developed for several Riemann solvers. A second order extension is also described.

In many physical situations, it is reasonable to assume that the relaxation is instantaneous: either the bubbles are very small or, more generally, the interfacial area between phases is large. We present an asymptotic expansion of the scheme 


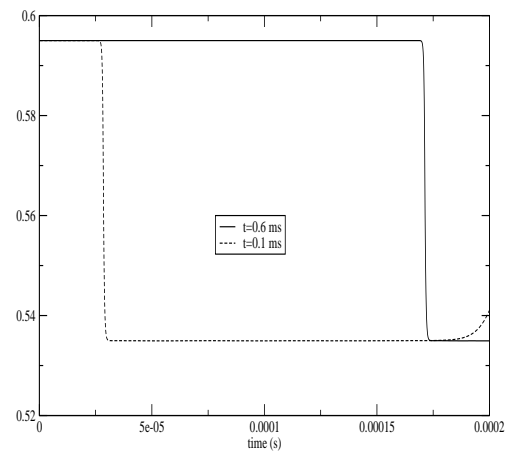

Volume fraction

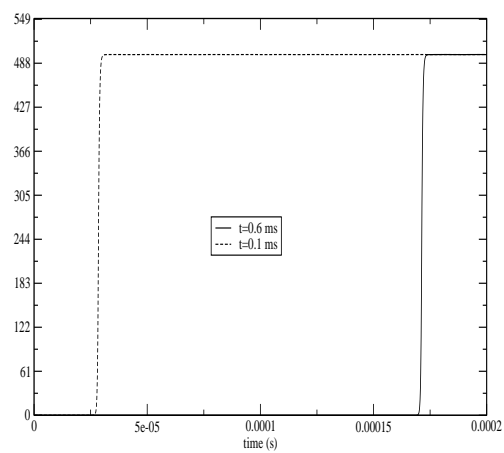

Velocity

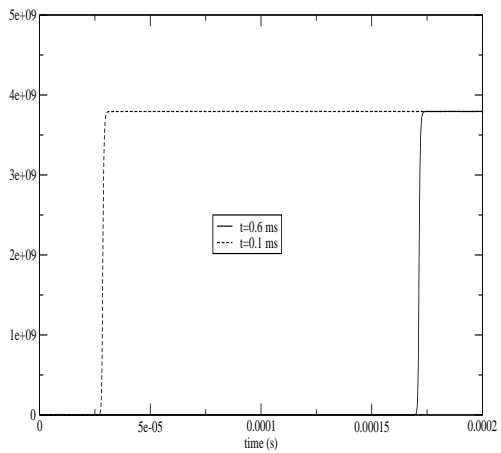

Pressure

FIG. 10. Evolution of the variables at $x=0.1 \mathrm{~m}$ and $x=0.6 \mathrm{~m}$ between the initial time and $t=2 \cdot 10^{-4} \mathrm{~s}$.

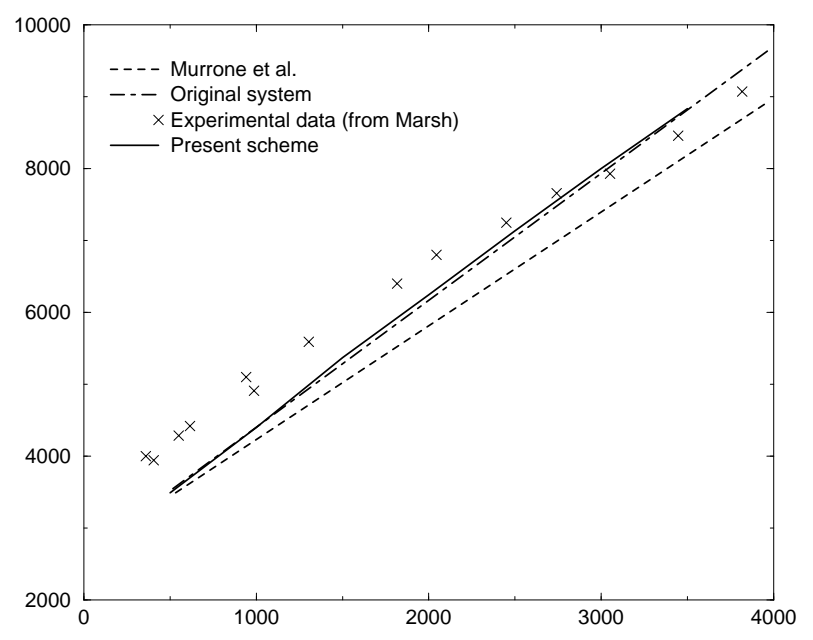

FIG. 11. Shock velocity, comparison between several approaches. 
that keeps the local conservation properties of the original scheme. The asymptotic expansion relies on the understanding of an equilibrium variety. Its structure depends, in principle, on the Riemann solver. We show that for several standard solvers that this is not the case, and hence this variety is characterized by the local pressure and velocity of the flow.

Several numerical test cases are presented in order to demonstrate the potential of this technique.

\section{REFERENCES}

[1] R. Abgrall, M. Papin, And L. Hallo, A scheme for compressible two-phases flows and interface problems, in Current Trends in Scientific Computing, Contemp. Math. 329, R. Glowinski, Z. (J.) Chen, and K. Li, eds., AMS, Providence, RI, 2003, pp. 1-12.

[2] R. Abgrall and R. Saurel, Discrete equations for physical and numerical compressible multiphase mixtures, J. Comput. Phys., 186 (2003), pp. 361-396.

[3] F. Coquel and B. Perthame, Relaxation of energy and approximate Riemann solvers for general pressure laws in fluid dynamics, SIAM J. Numer. Anal., 35 (1998), pp. 2223-2249.

[4] D. A. Drew and S. L. Passman, Theory of Multicomponent Fluids, Appl. Math. Sci. 135, Springer-Verlag, New York, 1999.

[5] S. EvJE AND K. FJELDE, Hybrid flux-splitting schemes for a two-phase flow model, J. Comput. Phys., 175 (2002), pp. 674-701.

[6] S. Marsh, LASL Shock Hugoniot Data, Los Alamos Series on Dynamic Material Properties, University of California Press, Berkeley, CA, 1980.

[7] R. Menikoff And B. Plohr, The Riemann problem for fluid flow of real materials, Rev. Modern Phys., 61 (1989), pp. 75-130.

[8] A. Murrone and H. Guillard, A five equation reduced model for compressible two phase flow problems, J. Comput. Phys., 202 (2005), pp. 664-698.

[9] R. D. Richtmyer And K. W. Morton, Difference Methods for Initial-Value Problems, 2nd ed., Interscience Tracts in Pure and Applied Mathematics 4, Interscience, John Wiley \& Sons, New York, London, Sydney, 1967.

[10] R. Saurel and R. Abgrall, Some models and methods for compressible multifluid and multiphase flows, J. Comput. Phys., 150 (1999), pp. 425-467.

[11] E. F. Toro, Riemann Solvers and Numerical Methods for Fluid Dynamics. A Practical Introduction, 2nd ed., Springer-Verlag, Berlin, 1999.

[12] I. Toumi, A weak formulation of Roe's approximate Riemann solver, J. Comput. Phys., 102 (1992), pp. 360-373. 